\title{
THE STEFAN PROBLEM WITH SMALL SURFACE TENSION
}

\author{
AVNER FRIEDMAN AND FERNANDO REITICH
}

\begin{abstract}
The Stefan problem with small surface tension $\varepsilon$ is considered. Assuming that the classical Stefan problem (with $\varepsilon=0$ ) has a smooth free boundary $\Gamma$, we denote the temperature of the solution by $\theta_{0}$ and consider an approximate solution $\theta_{0}+\varepsilon u$ for the case where $\varepsilon \neq 0, \varepsilon$ small. We first establish the existence and uniqueness of $u$, and then investigate the effect of $u$ on the free boundary $\Gamma$. It is shown that small surface tension affects the free boundary $\Gamma$ radically differently in the two-phase problem than in the one-phase problem.
\end{abstract}

\section{INTRODUCTION}

In the classical formulation of the two-phase Stefan problem the temperatures $\theta_{w}$ and $\theta_{i}$ of water and ice satisfy the following conditions on the interface $\{\Phi(x, t)=0\}$;

$$
\begin{gathered}
\nabla_{x} \theta_{w} \cdot \nabla_{x} \boldsymbol{\Phi}-\nabla_{x} \theta_{i} \nabla_{x} \boldsymbol{\Phi}=\Phi_{t}, \\
\theta_{w}=\theta_{i}=0
\end{gathered}
$$

the first condition is the conservation of energy. The functions $\theta_{w}, \theta_{i}$ further satisfy the heat equation in the water and ice sets, respectively, as well as initial and boundary conditions on the fixed portions of the boundary.

For definiteness we shall take in this paper the initial geometry to be as in Figure 1, namely, the fixed boundary $(\partial D)$ is surrounded by water (region $\left.G=G_{0} \backslash \bar{D}\right)$ and the water is surrounded by ice. The fixed boundary does not change in time, but the free boundary (the water-ice interface) will of course change with time.

The one-phase Stefan problem arises when $\theta_{i} \equiv 0$ in the ice region, or $\theta_{w} \equiv 0$ in the water region. Taking for definiteness the case $\theta_{i} \equiv 0$, the interface conditions are

$$
\nabla_{x} \theta_{w} \cdot \nabla_{x} \boldsymbol{\Phi}=\Phi_{t},
$$

Received by the editors October 20, 1989.

1980 Mathematics Subject Classification (1985 Revision). Primary 35R35; Secondary 35K20, $35 \mathrm{~K} 85$.

This work is partially supported by National Science Foundation Grant DMS-86-12880. 


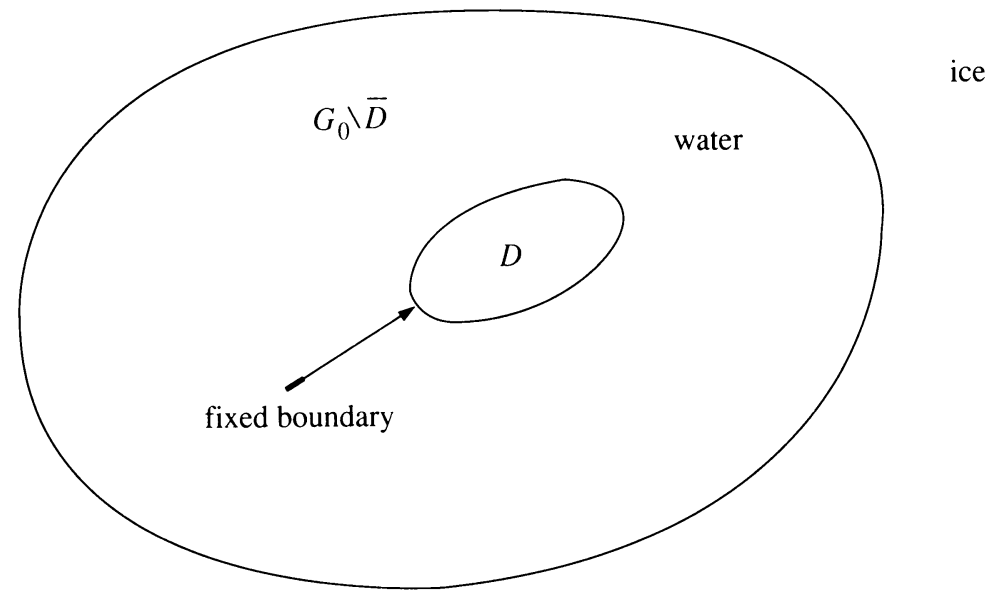

FIGURE 1

$$
\theta_{w}=0 \text {. }
$$

Molecular considerations attempting to explain dendritic growth of crystals suggest replacing $(0.4)$ by the Gibbs-Thomson relation

$$
\theta_{w}=\gamma \sigma \kappa
$$

where

(0.6) $\kappa=$ mean curvature of the interface $=\frac{\operatorname{div} \vec{\nu}}{n-1} \quad(n=$ dimension $)$,

$\vec{\nu}$ is the unit normal pointing into the ice (and extended as constant vector along each normal line), $\sigma$ is the surface tension and $\gamma$ is a positive constant; for details see $[1,2,8,12,17,18]$ and the references given there. Gurtin [9 and $10]$ has established $(0.5)$ and its counterpart

$$
\theta_{w}=\theta_{i}=\gamma \sigma \kappa
$$

for the two-phase case by thermodynamic considerations (see also Langer [14]). The sign of $\kappa$ in (0.6) is positive (negative) if the intersection of the water region (ice region) with a small ball centered at the interface point is convex.

It is well known that the Stefan problem with interface conditions $(0.1),(0.2)$ or (0.3), (0.4) has a unique global weak solution; see, for instance, [6]. Further, for the one-phase problem the free boundary is known to be smooth (for $t>0$ ) under some conditions on the initial geometry and the initial and boundary data (see [7, 6]); smoothness for large time only (i.e. for $t>t_{0}$ ) was established by Matano [15] under fairly weak assumptions on the data. For the 1- and 2-phase Stefan problems, smoothness of the free boundary was established by Meirmanov [16] and Hanzawa [11] for small time $(0 \leq t \leq \delta)$, provided the initial data are smooth and satisfy some compatibility conditions. 
On the other hand, for the Stefan problem with surface tension (i.e., (0.2) or $(0.4)$ are replaced by $(0.5)$ or $(0.7))$ no existence results are known; not even for small time. The nearest results in this direction are due to Duchon and Robert [3] for the one phase Stefan problem in two dimensions, whereby the heat equation $\theta_{t}-\Delta \theta=0$ is replaced by the Laplace equation $\Delta \theta=0$; they established local existence and uniqueness. Visintin [19] proved existence of a weak solution after replacing $(0.5)$ by another condition which is an approximation to the Gibbs-Thomson law.

Added in proof. Another version of a weak solution was studied recently by Luckhaus [20]; he proved existence of a solution.

In this paper we consider the Stefan problem with small surface tension $\sigma$ and linearize the problem about $\sigma=0$. The linearized problem turns out to be a nonstandard parabolic problem. We establish existence and uniqueness of a weak solution $u$ and then investigate the effect of $u$ on the original shape of the free boundary of the Stefan problem with zero surface tension.

The one-phase Stefan problem is considered in $\S \S 1-7$ and the two-phase Stefan problem is considered in $\S \S 8,9$.

In $\S 1$ we introduce the approximation $\theta=\theta_{0}+\gamma \sigma u$ and derive a linear parabolic problem for $u$; $\theta_{0}$ is the solution of the Stefan problem with $\sigma=0$. In $\S 2$ we give a weak formulation and in $\S 3$ it is proved that a weak solution $u$ exists. Uniqueness of the weak solution is established in $\S 4$. In $\S \S 6,7$ we investigate the perturbation of the free boundary of $\theta_{0}$ due to the term $u$. Assuming that the negative sign holds in (0.6) we prove that

small surface tension decreases

the water region, for all small times.

We also prove (and this is the main result of $\S \S 6,7)$ that

small surface tension increases the water region for all large times.

More precisely, the free boundary for large times is approximately of the form $|x|=M \sqrt{t} \quad(M>0)$ in the absence of surface tension and $|x|=M \sqrt{t}+\varepsilon M_{0}$ in the presence of surface tension ( $M$ and $M_{0}$ are positive constants).

Some ODE results needed in $\S \S 6,7$ are derived in $\S 5$.

In $\S \S 8,9$ we deal with the two-phase Stefan problem. In $\S 8$ we prove the existence and uniqueness of a weak solution $u=\left(u_{1}, u_{2}\right)$ such that $\theta_{0}+\varepsilon u$ is an approximate solution to the two-phase Stefan problem with small surface tension. In $\S 9$ we investigate the perturbation of the free boundary due to the term $u$. Assuming that the water region when $\sigma=0$ is convex, we find that $(0.8)$ is valid for all times; this is radically different (for large times) from the 1 -phase situation whereby $(0.9)$ holds.

Our results on existence and uniqueness of a solution to the linearized Stefan problem about $\sigma=0$ extend to geometries other than the one depicted in Figure 1. However the interesting conclusions on the effect of small surface tension on 
the free boundary (in $\S \S 6,7$ and 9 ) depend on the geometry of Figure 1, i.e., on the fact that the ice surrounds the water and the water surrounds the fixed boundary.

\section{THE APPROXIMATING SYSTEM}

Let $D$ be a bounded domain in $\mathbf{R}^{n}(n \geq 2)$ with $C^{2, \alpha}$ boundary $\partial D$. Let $G_{0}$ be a bounded domain in $\mathbf{R}^{n}$ with $C^{2, \alpha}$ boundary such that $\bar{D} \subset G_{0}$. Set $G=G_{0} \backslash \bar{D}$.

For any $T>0$, set $\partial D_{T}=\partial D \times(0, T]$. Consider the one-phase Stefan problem with surface tension:

$$
\begin{gathered}
\theta_{t}-\Delta \theta=0 \text { in } G_{T}, \\
\theta=\tilde{\theta} \quad \text { on } \partial D_{T} \cup(G \times\{0\}), \\
\theta=\varepsilon \kappa \quad \text { on the free boundary } \Gamma_{T}, \varepsilon>0, \\
\left(X_{t}+\nabla \theta\right) \cdot N=0 \text { on } \Gamma_{T} .
\end{gathered}
$$

Here $\Gamma_{T}=\bigcup_{0 \leq t \leq T} \Gamma(t)$ and for each $t \in[0, T]$, the free boundary $\Gamma(t)$ (i.e., the interface) is given by $x=X(s, t)$ where $s$ is $(n-1)$-dimensional parameter, $\kappa=\kappa(s, t)$ is the mean curvature of the surface $s \rightarrow X(s, t)$ at $s$, and $N=$ $N(s, t)$ is the outward unit normal of this surface. We easily find that

$$
(n-1) \kappa=\operatorname{div}_{x} N=\text { trace of } M(s, t)
$$

where $M(s, t)$ is the matrix in $\mathbf{R}^{n \times n}$ defined by the relations

$$
M N=0, \quad M X_{s_{j}}=N_{s_{j}} \quad(j=1, \ldots, n-1) .
$$

In (1.3) $\varepsilon=\gamma \sigma$ where $\gamma, \sigma$ are as in (0.5).

In the sequel, the initial and boundary data, designated by $\tilde{\theta}$ in (1.2), are assumed to be positive valued on $\partial D_{T} \cup(G \times\{0\})$, and $\tilde{\theta}(x, 0)=0$ on $\partial G_{0}$.

The water region at time $t$ is designated by $G(t)$, and $G_{T}=\bigcup_{0 \leq t \leq T} G(t)$; note that $\partial G(t)=\partial D(t) \cup \Gamma(t)$ where $\partial D(t)=\partial D \times\{t\}$.

Existence theorems for (1.1)-(1.4) have not been established up to now.

Consider next the Stefan problem with zero surface tension:

$$
\begin{gathered}
\theta_{t}-\Delta \theta=0 \text { in } G_{T}, \\
\theta=\tilde{\theta} \text { on } \partial D_{T} \cup(G \times\{0\}), \\
\theta=0 \text { on the free boundary } \Gamma_{T}, \\
\left(z_{t}+\nabla \theta\right) \cdot N_{0}=0 \text { on } \Gamma_{T}
\end{gathered}
$$

where $\Gamma_{T}=\bigcup_{0 \leq t \leq T} \Gamma(t)$ and, for each $t$, the free boundary $\Gamma(t)$ is given by

$$
s \rightarrow x=z(s, t), \quad s=\left(s_{1}, \ldots, s_{n-1}\right), \quad 0 \leq s_{i} \leq L_{i} ;
$$

thus $z(s, t)$ is defined in the rectangle

$$
L=\prod_{i=1}^{n-1}\left\{0 \leq s_{i} \leq L_{i}\right\}
$$


and is $L_{i}$-periodic in $s_{i}$ for each $i$. The vector $N_{0}$ in (1.8) is an exterior normal, and $G_{T}=\bigcup_{0 \leq t \leq T} G(t)$, where $G(t)$ is the region occupied by water.

From the results of Hanzawa [11] and Meirmanov [16] it follows that a solution of $(1.5)-(1.9)$ exists for small $T$ and $\Gamma_{T} \in C^{j+\beta}$ provided

$$
\begin{aligned}
& \tilde{\theta}(x, 0) \text { and } \partial G_{0} \text { are sufficiently smooth, say } \\
& \text { in } C^{m_{j}+\beta} \text {, and } \tilde{\theta}(x, 0) \text { satisfies the compatibility } \\
& \text { conditions of order } m_{j}-2 \text { at } \partial G_{0} .
\end{aligned}
$$

As for global existence of smooth solutions, the following result was proved by Friedman and Kinderlehrer [7]:

Suppose $D$ is a ball $\left\{|x|<r_{0}\right\}$ and $G_{0}$ is star-shaped with respect to any point in $\left\{|x|<\delta_{0}\right\}$ for some $0<\delta_{0}<r_{0}$. Using polar coordinates $\left(r, \theta_{1}, \ldots, \theta_{n-1}\right)$ about 0 , write

$$
\Delta v=r^{1-n}\left(r^{n-1} v_{r}\right)_{r}+r^{-2} A v
$$

and assume that

$$
\begin{gathered}
\left(r^{n-1} \tilde{\theta}(x, 0)\right)_{r}<0, \quad x \in \bar{G}, \\
\tilde{\theta}(x, t)-\tilde{\theta}(x, 0)-\frac{1}{r_{0}^{2}} A\left(\int_{0}^{t} \tilde{\theta}(x, s) d s\right)>0, \quad|x|=r_{0}, \quad 0<t \leq T .
\end{gathered}
$$

Thus the solution of (1.5)-(1.9) exists and the free boundary is given by

$$
r=\rho\left(\theta_{1}, \ldots, \theta_{n-1}, t\right), \quad 0 \leq t \leq T
$$

where

$$
\rho \in C^{\infty} \quad \text { in }\left(\theta_{1}, \ldots, \theta_{n-1}, t\right), \quad 0<t \leq T .
$$

By combining the local and global results we obtain a solution of (1.5)-(1.8) with the boundary given by $(1.10)$ such that

$$
\begin{gathered}
\rho \in C^{j+\beta} \quad \text { if } 0 \leq t \leq t^{*} \text { for some small } t^{*}>0, \\
\quad \rho \in C^{\infty} \quad \text { if } 0<t \leq T .
\end{gathered}
$$

We shall henceforth denote the solution to $(1.5)-(1.8)$ by $\left(\theta_{0}, z\right)$; the free boundary will be denoted by $\Gamma_{T}$ and the water region $\left\{\theta_{0}>0\right\}$ by $G_{T}$. We shall always assume that $z \in C^{3+\beta}$ for $s \in L, 0 \leq t \leq T$.

We shall try to find an approximate solution to (1.1)-(1.4) of the form

$$
\theta=\theta_{0}+\varepsilon u
$$

with free boundary

$$
x=z(s, t)+\varepsilon \zeta(s, t) .
$$

We shall choose an outward normal $N=N_{0}$ to $x=z(s, t)$ and an outward normal $N=N_{0}+\varepsilon N_{1}$ to $x=z+\varepsilon \zeta$ as follows: $N_{0}$ has components

$$
N_{0, i}=(-1)^{n+i}\left|\begin{array}{cccccc}
\frac{\partial z_{1}}{\partial s_{1}} & \cdots & \frac{\partial z_{i-1}}{\partial s_{1}} & \frac{\partial z_{i+1}}{\partial s_{1}} & \cdots & \frac{\partial z_{n}}{\partial s_{1}} \\
\vdots & & \vdots & \vdots & & \vdots \\
\frac{\partial z_{1}}{\partial s_{n-1}} & \cdots & \frac{\partial z_{i-1}}{\partial s_{n-1}} & \frac{\partial z_{i+1}}{\partial s_{n-1}} & \cdots & \frac{\partial z_{n}}{\partial s_{n-1}}
\end{array}\right|
$$


and $N_{1}$ has components $N_{1, i}$ obtained from

$$
\tilde{N}_{1, i}(\varepsilon)=(-1)^{n+i}\left|\begin{array}{cccccc}
\frac{\partial\left(z_{1}+\varepsilon \zeta_{1}\right)}{\partial s_{1}} & \ldots & \frac{\partial\left(z_{i-1}+\varepsilon \zeta_{i-1}\right)}{\partial s_{1}} & \frac{\partial\left(z_{i+1}+\varepsilon \zeta_{i+1}\right)}{\partial s_{1}} & \ldots & \frac{\partial\left(z_{n}+\varepsilon \zeta_{n}\right)}{\partial s_{1}} \\
\vdots & & \vdots & \vdots & & \vdots \\
\frac{\partial\left(z_{1}+\varepsilon \zeta_{1}\right)}{\partial s_{n-1}} & \ldots & \frac{\partial\left(z_{i-1}+\varepsilon \zeta_{i-1}\right)}{\partial s_{n-1}} & \frac{\partial\left(z_{i+1}+\varepsilon \zeta_{i+1}\right)}{\partial s_{n-1}} & \ldots & \frac{\partial\left(z_{n}+\varepsilon \zeta_{n}\right)}{\partial s_{n-1}}
\end{array}\right|
$$

by $N_{1, i}=\left.\frac{d}{d \varepsilon} \tilde{N}_{1, i}(\varepsilon)\right|_{\varepsilon=0}$.

One can easily deduce the structure

$$
N_{1}=\sum_{j=1}^{n-1} A_{j} \frac{\partial \zeta}{\partial s_{j}}
$$

where $A_{j}$ are matrices with elements which depend on $\partial z_{i} / \partial s_{k}$ in general. From (1.3) we get

$$
\theta_{0}(z+\varepsilon \zeta, t)+\varepsilon u(z+\varepsilon \zeta, t)=\varepsilon \kappa+O\left(\varepsilon^{2}\right)
$$

in view of $(1.7)$, this gives

$$
\nabla \theta_{0} \cdot \zeta+u=\kappa \quad \text { on } x=z(s, t) .
$$

Next, from (1.4) we have

$$
\left[z_{t}+\varepsilon \zeta_{t}+\nabla \theta_{0}(z+\varepsilon \zeta, t)+\varepsilon \nabla u(z+\varepsilon \zeta, t)\right] \cdot\left(N_{0}+\varepsilon N_{1}\right)=O\left(\varepsilon^{2}\right) .
$$

Recalling (1.8) we get

$$
\begin{aligned}
\left(z_{t}+\right. & \left.\nabla \theta_{0}\right) \cdot N_{1}+\zeta \cdot N_{0}+\nabla u \cdot N_{0} \\
& +\left(\nabla \partial_{x_{1}} \theta_{0} \cdot \zeta, \ldots, \nabla \partial_{x_{n}} \theta_{0} \cdot \zeta\right) \cdot N_{0}=0 \quad \text { on } x=z(s, t)
\end{aligned}
$$

In equations (1.13), (1.14), the mean curvature $\kappa$ of $s \rightarrow z(s, t)$ is known, $N_{0}$ and $\nabla \theta_{0}$ are also known, but $u$ and $\zeta$ are unknown (by (1.12) $N_{1}$ is known once $\zeta$ is known). We wish to eliminate $\zeta$, so as to obtain a single relation for $u$ on the free boundary $\Gamma_{T}: x=z(s, t)$. To do this, we write $\zeta$ in the form

$$
\zeta(s, t)=R(s, t) N_{0}(s, t) .
$$

Substituting this into (1.13) we get

$$
R(s, t)=\frac{\kappa-u}{\nabla \theta_{0} \cdot N_{0}}=h_{0}(u-\kappa), \quad h_{0}=-\frac{1}{\nabla \theta_{0} \cdot N_{0}} \geq m>0,
$$

where $m$ is a constant; the positivity of $h_{0}$ follows from the maximum principle applied to $\theta_{0} \quad\left(\theta_{0}\right.$ takes minimum at the free boundary).

Substituting $\zeta$ from (1.15) into (1.14) and recalling (1.12), we get

$$
\begin{array}{r}
\left(z_{t}+\nabla \theta_{0}\right) \cdot \sum A_{j}\left(R_{s_{j}} N_{0}+R N_{0, s_{j}}\right)+N_{0} \cdot R_{t} N_{0}+N_{0} \cdot R N_{0, t} \\
+\nabla u \cdot N_{0}+N_{0} \cdot\left(\nabla \partial_{x_{1}} \theta_{0} \cdot R N_{0}, \ldots, \nabla \partial_{x_{n}} \theta_{0} \cdot R N_{0}\right)=0
\end{array}
$$


or

$$
\begin{aligned}
& N_{0} \cdot N_{0} R_{t}+\sum\left(z_{t}+\nabla \theta_{0}\right) \cdot\left(A_{j} N_{0}\right) R_{s_{j}} \\
& \quad+\left\{\left(z_{t}+\nabla \theta_{0}\right) \cdot \sum A_{j} N_{0, s_{j}}+N_{0} \cdot N_{0, t}\right. \\
& \left.\quad+N_{0} \cdot\left(\nabla \partial_{x_{1}} \theta_{0} \cdot N_{0}, \ldots, \nabla \partial_{x_{n}} \theta_{0} \cdot N_{0}\right)\right\} R+\nabla u \cdot N_{0}=0 .
\end{aligned}
$$

Substituting $R$ from (1.16) into (1.17), we obtain

$$
\begin{aligned}
N_{0} \cdot & N_{0} \frac{d}{d t}\left(h_{0} u-h_{0} \kappa\right)+\left(z_{t}+\nabla \theta_{0}\right) \cdot \sum A_{j} N_{0} \frac{d}{d s_{j}}\left(h_{0} u-h_{0} \kappa\right) \\
+ & \left\{\left(z_{t}+\nabla \theta_{0}\right) \cdot \sum A_{j} N_{0, s_{j}}+N_{0} \cdot N_{0, t}\right. \\
& \left.\quad+N_{0} \cdot\left(\nabla \partial_{x_{1}} \theta_{0} \cdot N_{0}, \ldots, \nabla \partial_{x_{n}} \theta_{0} \cdot N_{0}\right)\right\}\left(h_{0} u-h_{0} \kappa\right) \\
& +\nabla u \cdot N_{0}=0 \quad \text { on } x=z(s, t) .
\end{aligned}
$$

The function $u$ also satisfies

$$
\begin{gathered}
u_{t}-\Delta u=0 \text { in } G_{T}, \\
u=0 \quad \text { on } \partial D_{T} \text { and on } G \times\{0\} .
\end{gathered}
$$

If $u$ is a solution to (1.18)-(1.20), then defining $\zeta$ by (1.15) where $R$ is given by (1.16), the pair

$$
\theta=\theta_{0}+\varepsilon u, \quad x=z+\varepsilon \zeta
$$

will form a first oráer approximation to problem (1.1)-(1.4).

We wish to study the linear problem $(1.18)-(1.20)$ and to analyze the effect of the term $u$ on the free boundary $\Gamma_{T}$. Note that (1.18) can be written in the form

$$
a \frac{d u}{d t}+\sum b_{j} \frac{d u}{d s_{j}}+N_{0} \cdot \nabla u+c u=f_{0}
$$

where

$$
\begin{aligned}
a= & N_{0} \cdot N_{0} h_{0}, \quad b_{j}=\left(z_{t}+\nabla \theta_{0}\right) \cdot A_{j} N_{0} h_{0}, \\
c= & N_{0} \cdot N_{0} \frac{d h_{0}}{d t}+\left(z_{t}+\nabla \theta_{0}\right) \cdot \sum_{j} A_{j} N_{0} \frac{d h_{0}}{d s_{j}} \\
+ & \left\{\left(z_{t}+\nabla \theta_{0}\right) \cdot \sum_{j} A_{j} N_{0, s_{j}}+N_{0} \cdot N_{0, t}\right. \\
& \left.+N_{0} \cdot\left(\nabla \partial_{x_{1}} \theta_{0} \cdot N_{0}, \ldots, \nabla \partial_{x_{n}} \theta_{0} \cdot N_{0}\right)\right\} h_{0}, \\
f_{0}= & N_{0} \cdot N_{0} \frac{d}{d t}\left(h_{0} \kappa\right)+\left(z_{t}+\nabla \theta_{0}\right) \cdot \sum A_{j} N_{0} \frac{d}{d s_{j}}\left(h_{0} \kappa\right) \\
+ & \left\{\left(z_{t}+\nabla \theta_{0}\right) \cdot \sum A_{j} N_{0, s_{j}}+N_{0} \cdot N_{0, t}\right. \\
& \left.+N_{0} \cdot\left(\nabla \partial_{x_{1}} \theta_{0} \cdot N_{0}, \ldots, \nabla \partial_{x_{n}} \theta_{0} \cdot N_{0}\right)\right\} h_{0} \kappa .
\end{aligned}
$$


$\operatorname{In}(1.22)$

$$
\begin{gathered}
u=u(z(s, t), t) \quad \text { and } \frac{d u}{d t}=\sum_{i=1}^{n} \frac{\partial u}{\partial x_{i}} \frac{\partial z_{i}}{\partial t}+\frac{\partial u}{\partial t} \\
\frac{d u}{d s_{j}}=\sum_{i=1}^{n} \frac{\partial u}{\partial x_{i}} \frac{\partial z_{i}}{\partial s_{j}} .
\end{gathered}
$$

Similarly one understands the expressions $d h_{0} / d t, d h_{0} / d s$, etc. in (1.23).

Remark 1.1. Since $z \in C^{3+\beta}$, a belongs to $C^{2+\beta}, b_{j}$ belongs to $C^{1+\beta}$, and $c, f_{0}$ belong to $C^{\beta}$. For the results of $\S \S 2-4$ it actually suffices that $c, f_{0}$ are continuous functions and $a, b_{j}$ are continuously differentiable.

\section{Definition OF WEAK SOLUTION}

In $\S 1$ we derived for $u=[\partial \theta / \partial \varepsilon]_{\varepsilon=0}$ the parabolic system

$$
\begin{gathered}
u_{t}-\Delta u=0 \text { in } G_{T}, \\
u=0 \text { on } \partial D_{T} \text { and on } G \times\{0\}, \\
a \frac{d u}{d t}+\sum_{j=1}^{n-1} b_{j} \frac{d u}{d s_{j}}+N_{0} \cdot \nabla u+c u=f_{0} \quad \text { on } \Gamma_{T}
\end{gathered}
$$

where $a, b_{j}, c, f_{0}$ are defined in $(1.23)$;

$$
a \geq a_{0}>0 \quad\left(a_{0} \text { constant }\right) .
$$

The boundary condition (2.3) is nonstandard. To prove existence (and uniqueness) we shall resort to working with a weak formulation of (2.1)-(2.3).

Let $\varphi(x, t)$ be any smooth function such that $\varphi=0$ on $\partial D_{T}$ and on $G(T)$. Formally,

$$
\begin{aligned}
0 & =\int_{G_{T}} \varphi\left(u_{t}-\Delta u\right)=\int_{0}^{T} \int_{G(t)} \varphi\left(u_{t}-\Delta u\right) d x d t \\
& =\int_{G(T)} d x \int_{t(x)}^{T} \varphi u_{t} d t-\int_{0}^{T} \int_{\Gamma(t)} \varphi u_{\nu} d \sigma_{t} d t+\int_{0}^{T} \int_{G(t)} \nabla \varphi \cdot \nabla u
\end{aligned}
$$

where $\nu$ is the outward unit normal and $t(x)=\min \left\{t_{0} ;\left(x, t_{0}\right) \in G_{T}\right\}$ (recall that the sets $G(t)$ increase with $t)$. Observe that by definition of the surface area $d \sigma_{t}$, on $\Gamma(t), d \sigma_{t}=\left\|N_{0}\right\| d s$. Hence we get

$$
\begin{aligned}
0= & -\int_{G(T)} d x \int_{t(x)}^{T} \varphi_{t} u-\int_{G(T) \backslash G(0)}(\varphi u)(x, t(x)) d x \\
& +\int_{G_{T}} \nabla \varphi \cdot \nabla u-\int_{0}^{T} \int_{L} \varphi u_{\nu}\left\|N_{0}\right\| d s d t
\end{aligned}
$$

and $u_{\nu}\left\|N_{0}\right\|=\nabla u \cdot N_{0}$. Also

$$
\int_{G(T) \backslash G(0)}(\varphi u)(x, t(x)) d x=\int_{0}^{T} \int_{L}(\varphi u)(z(s, t), i)\left|\frac{\partial z(s, t)}{\partial(s, t)}\right| d s d t
$$


by the change of variables $x \rightarrow z(s, t)$. It follows that

$$
\begin{gathered}
-\int_{G_{T}} u \varphi_{t}+\int_{G_{T}} \nabla \varphi \cdot \nabla u-\int_{0}^{T} \int_{L} u(z(s, t), t) \varphi(z(s, t), t)\left|\frac{\partial z(s, t)}{\partial(s, t)}\right| \\
-\int_{0}^{T} \int_{L} \varphi(z(s, t), t) \nabla u \cdot N_{0} d s d t=0 .
\end{gathered}
$$

In the last integral we substitute $\nabla u \cdot N_{0}$ from (2.3) and integrate by parts in the integrals

$$
\iint a \frac{d u}{d t} \varphi, \quad \iint b_{j} \frac{d u}{d s_{j}} \varphi .
$$

We then obtain from $(2.5)$

$$
\begin{aligned}
& -\int_{G_{T}} u \varphi_{t}+\int_{G_{T}} \nabla \varphi \cdot \nabla u \\
& \quad-\int_{0}^{T} \int_{L} u(z(s, t), t)\left\{\frac{d}{d t}(a \varphi)+\sum \frac{d}{d s_{j}}\left(b_{j} \varphi\right)-c \varphi+\left|\frac{\partial z(s, t)}{\partial(s, t)}\right| \varphi\right\} d s d t \\
& =\int_{0}^{T} \int_{L} \varphi f_{0} d s d t .
\end{aligned}
$$

Set

$$
\mathscr{A}_{T}=\left\{\varphi \in C^{1}\left(\overline{G_{T}}\right), \varphi=0 \text { on } G(T) \cup \partial D_{T}\right\}
$$

Definition 2.1. A function $u$ is a weak solution of (2.1)-(2.3) if

$$
u, \nabla u \in L^{2}\left(G_{T}\right)
$$

(2.6) holds for any $\varphi \in \mathscr{A}_{T}$, and $u=0$ on $\partial D_{T}$ and on $G \times\{0\}$ in the usual continuous sense.

Notice that, by $(2.7), u \in L^{2}\left(\Gamma_{T}\right)$.

Notice also that (2.6) implies that (2.1) holds in $G_{T}$ and therefore $u$ is a smooth function away from the free boundary $\Gamma_{T}$.

\section{EXISTENCE OF WEAK SOLUTION}

The existence of a weak solution depends upon an energy inequality. We first proceed to derive this inequality in a formal manner, assuming that $u$ is smooth up to $\Gamma_{T}$ (and thus it satisfies (2.3)). Multiplying (2.1) by $u$ and integrating 
over $G_{\tau} \equiv G_{T} \cap\{t \leq \tau\} \quad(\tau \in(0, T))$ we get

$$
\begin{aligned}
0= & 2 \int_{G_{\tau}} u\left(u_{t}-\Delta u\right)=\int_{G(\tau)} \int_{t(x)}^{\tau}\left(u^{2}\right)_{t} d t d x \\
& +2 \int_{G_{\tau}}|\nabla u|^{2}-2 \int_{0}^{\tau} \int_{\partial G(t)} u u_{\nu} d \sigma_{t} d t \\
= & \int_{G(\tau)} u^{2}(x, \tau) d x-\int_{G(\tau) \backslash G(0)} u^{2}(x, t(x)) d x+2 \int_{G_{\tau}}|\nabla u|^{2} \\
& -2 \int_{0}^{\tau} \int_{L} u\left(\nabla u \cdot N_{0}\right) d s d t \\
= & \int_{G(\tau)} u^{2}(x, \tau) d x+2 \int_{G_{\tau}}|\nabla u|^{2}-\int_{0}^{t} \int_{L} u^{2}\left|\frac{\partial z}{\partial(s, t)}\right|^{2} \\
& +2 \int_{0}^{t} \int_{L} u\left(a \frac{d u}{d t}+\sum b_{j} \frac{d u}{d s_{j}}+c u-f_{0}\right)
\end{aligned}
$$

by (2.3). Hence, after integration by parts in the last integral,

$$
\begin{aligned}
\int_{G(\tau)} u^{2}+2 \int_{G_{\tau}}|\nabla u|^{2}+\int_{L} a(z(s, \tau), \tau) u^{2}(z(s, \tau), \tau) d s \\
=\int_{0}^{\tau} \int_{L} u^{2}\left[\left|\frac{\partial z}{\partial(s, t)}\right|+\frac{d a}{d t}+\sum_{j=1}^{n-1} \frac{d b_{j}}{d s_{j}}-2 c\right] d s d t \\
+\int_{0}^{\tau} \int_{L} u f_{0} d s d t
\end{aligned}
$$

where the argument in each function in the integrands on the right-hand side is $(z(s, t), t)$. Since the right-hand side of $(3.1)$ is bounded by

$$
C \int_{0}^{\tau} \int_{L} u^{2}+\int_{0}^{\tau} \int_{L} f_{0}^{2},
$$

we obtain, after using Gronwall's inequality, the desired energy inequality

$$
\begin{gathered}
\sup _{0<t<T} \int_{G(t)} u^{2}(x, t) d x+\int_{G_{T}}|\nabla u|^{2} d x d t \\
\quad+\sup _{0<t<T} \int_{L} u^{2}(z(s, t), t) d s \leq C_{T} .
\end{gathered}
$$

The strategy for constructing a weak solution is to first work with a finitedifference scheme, establish existence and an energy-type inequality analogous to (3.2), and then pass to the limit.

We shall use the finite differences

$$
\begin{gathered}
u_{t}^{-}(x, k)=\frac{1}{h}(u(x, k h)-u(x,(k-1) h)) \quad(\text { backward }), \\
u_{t}(x, k)=\frac{1}{h}(u(x,(k+1) h)-u(x, k h)) \quad(\text { forward })
\end{gathered}
$$


where $h$ is any positive number. We introduce a finite-difference version of (2.1)-(2.3):

$$
\begin{gathered}
u_{t}^{-}-\Delta u=0 \quad \text { in } G(k h) \equiv G^{k} \\
a \frac{1}{h}[u(z(s, k h), k h)-u(z(s,(k-1) h),(k-1) h)] \\
+\sum b_{j} \frac{d u}{d s_{j}}+N_{0} \cdot \nabla u+c u=f_{0} \quad \text { on } \Gamma(k h) \equiv \Gamma^{k} \\
u=0 \quad \text { on } \partial D(k h) \equiv \partial D^{k}
\end{gathered}
$$

for $k=1, \ldots, m$, where $T-h<m h \leq T$. If we set

$$
u^{k}=u(x, k h), \quad a^{k}=a(x, k h), \text { etc. }
$$

then (3.3)-(3.5) read

$$
\frac{1}{h} u^{k}-\Delta u^{k}=\frac{1}{h} u^{k-1} \text { in } G^{k}
$$

$$
\begin{gathered}
\frac{1}{h} a^{k} u^{k}+N_{0}^{k} \cdot \nabla u^{k}+\sum b_{j}^{k} \frac{d u^{k}}{d s_{j}}+c^{k} u^{k}=\frac{1}{h} a^{k} u^{k-1}+f_{0}^{k} \text { on } \Gamma^{k}, \\
u^{k}=0 \text { on } \partial D^{k} .
\end{gathered}
$$

Since $u^{k-1}$ is defined only in $G^{k-1}$ and $G^{k-1} \subset G^{k}$, before we can study the system $\left(3.3_{k}\right)-\left(3.5_{k}\right)$ we must extend the definition of $u^{k-1}$ into all of $G^{k} \backslash G^{k-1}$. We define this extension using the boundary values of $u^{k-1}$ :

$$
\begin{aligned}
& u^{k-1}(z(s, t))=u^{k-1}(z(s,(k-1) h),(k-1) h) \\
& \text { for } s \in L,(k-1) h<t<k h .
\end{aligned}
$$

Thus, for each $s \in L, u^{k-1}(x)$ is constant along the curve $t \rightarrow z(s, t)$ passing through $(x, t(x))$.

The system $\left(3.3_{k}\right)-\left(3.5_{k}\right)$ is now a well defined elliptic problem for $u^{k}$. Since the boundary condition $\left(3.4_{k}\right)$ has the form

$$
\alpha_{0} \frac{\partial u}{\partial \nu}+\sum \alpha_{j} \frac{\partial u^{k}}{\partial s_{j}}+\beta u^{k}=\gamma
$$

where $\nu$ is the outward unit normal and $\alpha_{0}, \beta$ are positive functions, (if $h$ is sufficiently small), the system $\left(3.3_{k}\right)-\left(3.5_{k}\right)$ (or equivalently $(3.3)-(3.5)$ ) has a unique solution with $u(x, 0)=0$.

We proceed to derive an energy inequality for the $u^{k}$, s, analogous to (3.2). We shall need the identity [13, p. 246]

$$
2 \alpha_{r} u_{r}\left(u_{r}-u_{r-1}\right)=\alpha_{r} u_{r}^{2}-\alpha_{r-1} u_{r-1}^{2}-\left(\alpha_{r}-\alpha_{r-1}\right) u_{r-1}^{2}+\alpha_{r}\left(u_{r}-u_{r-1}\right)^{2}
$$


Taking $\alpha_{r}=a^{r}$ we get

$$
\begin{aligned}
2 h \sum_{k=1}^{k_{0}} a^{k} u^{k} u_{t}^{-}(k)= & a^{k_{0}}\left(u^{k_{0}}\right)^{2}-a^{0}\left(u^{0}\right)^{2}-h \sum_{k=0}^{k_{0}-1} a_{t}(k)\left(u^{k}\right)^{2} \\
& +h^{2} \sum_{k=1}^{k_{0}} a^{k}\left(u_{t}^{-}(k)\right)^{2}
\end{aligned}
$$

where $u_{t}^{-}(k)$ is the function $u_{t}^{-}(x, k)$, and $a_{t}(k)$ is $a_{t}(x, k)$.

Set

$$
\lambda(x)=\min \left\{j ; x \in G^{j}\right\}
$$

Multiplying $\left(3.3_{k}\right)$ by $u^{k}$, integrating over $G^{k}$ and then summing over $k$, $1 \leq k \leq k_{0}$, we get

$$
\begin{aligned}
0= & 2 h \sum_{k=1}^{k_{0}} \int_{G^{k}} u_{t}^{-} u-2 h \sum_{k=1}^{k_{0}} \int_{G^{k}} u \Delta u \\
= & \left.\int_{G^{k_{0}}} 2 h \sum_{k=\lambda(x)}^{k_{0}} u_{t}^{-} u-2 h \sum_{k=1}^{k_{0}} \int_{G^{k}} u \Delta u \quad \text { by (3.8) with } a \equiv 1\right) \\
= & \int_{G^{k_{0}}} u^{2}\left(x, k_{0} h\right)-\int_{G^{k_{0}}} u^{2}(x,(\lambda(x)-1) h)+h^{2} \int_{G^{k_{0}}} \sum_{k=\lambda(x)}^{k_{0}}\left(u_{t}^{-}\right)^{2} \\
& -2 h \sum_{k=1}^{k_{0}} \int_{\partial G^{k}} u \frac{\partial u}{\partial \nu}+2 h \sum_{k=1}^{k_{0}} \int_{G^{k}}|\nabla u|^{2} .
\end{aligned}
$$

The second integral on the right-hand side can be written in the form

$$
\begin{aligned}
& \sum_{k=1}^{k_{0}} \int_{G^{k} \backslash G^{k-1}} u^{2}(x,(k-1) h) d x \\
& \quad=\sum_{k=1}^{k_{0}} \int_{(k-1) h}^{k h} \int_{L} u^{2}(z(s,(k-1) h),(k-1) h)\left|\frac{\partial z(s, t)}{\partial(s, t)}\right| d s d t
\end{aligned}
$$

(by (3.6) and change of variables).

Also,

$$
\int_{\partial G^{k}} u \frac{\partial u}{\partial \nu}=\int_{L} u^{k} \nabla u^{k} \cdot N_{0}^{k} d s
$$

and in the last integrand we can substitute $\nabla u^{k} \cdot N_{0}^{k}$ from $\left(3.4_{k}\right)$. Using these 
remarks, we can transform the right-hand side of (3.9) to obtain

$$
\begin{aligned}
0= & \int_{G^{k_{0}}} u^{2}\left(x, k_{0} h\right)+2 h \sum_{k=1}^{k_{0}} \int_{G^{k}}|\nabla u|^{2}+h^{2} \int_{G^{k_{0}}} \sum_{k=\lambda(x)}^{k_{0}}\left(u_{t}^{-}\right)^{2} \\
& -\sum_{k=1}^{k_{0}} \int_{L}\left[u^{k}(z(s,(k-1) h)]^{2} \int_{(k-1) h}^{k h}\left|\frac{\partial z(s, t)}{\partial(s, t)}\right| d s d t\right. \\
& +\left.\int_{L} a u^{2}(z(s, t), t) d s\right|_{t=0} ^{t=k_{0} h} \\
& -h \sum_{k=0}^{k_{0}-1} \int_{L} a u^{2} d s+h^{2} \sum_{k=1}^{k_{0}} \int_{L}\left(u_{t}^{-}\right)^{2} a d s \\
& -h \sum_{k=1}^{k_{0}} \int_{L} u^{2} \sum \frac{d b_{j}}{d s_{j}}+2 h \sum_{k=1}^{k_{0}} \int_{L} c u^{2} d s-2 h \sum_{k=1}^{k_{0}} \int_{L} u f_{0} .
\end{aligned}
$$

Notice that in the last five integrals the integrand $u$ is evaluated at $z(s, k h)$; the same applies to $a, b_{j}, c, f_{0}$ and the finite difference $a_{t}$. In deriving (3.10) we have used (3.8) in order to "integrate by parts" the expression

$$
\sum_{k=1}^{k_{0}} \int_{L} a u u_{t}^{-} d s
$$

From (3.10) we get

$$
\int_{G^{k_{0}}} u^{2}\left(x, k_{0} h\right)+\int_{L} a u^{2}\left(z\left(s, k_{0} h\right), k_{0} h\right) d s+2 h \sum_{k=1}^{k_{0}} \int_{G^{k}}|\nabla u|^{2} \leq-J
$$

where $J$ represents all the remaining terms in (3.10) with the exception of

$$
h^{2} \int_{G^{k_{0}}} \sum\left(u_{t}^{-}\right)^{2}+h^{2} \sum \int_{L}\left(u_{t}^{-}\right)^{2} a
$$

which have been dropped. It is easily seen that

$$
|J| \leq C h \sum_{k=1}^{k_{0}} \int_{L} u^{2}(z(s, k h), k h) d s+h \sum_{k=1}^{k_{0}} \int_{L} f_{0}^{2} .
$$

Substituting this into (3.11) and using Gronwall's inequality, we find that

$$
\begin{gathered}
\sup _{1 \leq k_{0} \leq m} \int_{G^{k_{0}}} u^{2}\left(x, k_{0} h\right) d x+h \sum_{k=1}^{m} \int_{G^{k}}|\nabla u|^{2} d x \\
+\sup _{1 \leq k_{0} \leq m} \int_{L} u^{2}\left(z\left(s, k_{0} h\right), k_{0} h\right) d s \leq C_{T}
\end{gathered}
$$

where $C_{T}$ is a constant independent of $h$. This inequality is analogous to (3.2).

Define a function $u^{h}$ by

$$
u^{h}(x, t)=u(x, k h) \text { for } x \in G^{k+1}, t \in[k h,(k+1) h) .
$$


Denote by $W$ the completion of the set of smooth functions $\psi$ which vanish on $G \times\{0\}$ and on $\partial D_{T}$ with the respect to the norm

$$
\|\psi\|=\int_{G_{T}}\left(\psi^{2}+\left|\nabla_{x} \psi\right|^{2}\right) ;
$$

$W$ is a Hilbert space.

From (3.12) we conclude that there exists a sequence $u^{h}$ with $h=h_{j} \rightarrow 0$ which is weakly convergent to a function $u$ in $W$; we can choose the $h$ 's so that $T / h=m, m$ an integer

Theorem 3.1. The function $u$ is a weak solution of (2.1)-(2.3).

Proof. It suffices to show that (2.6) is satisfied for any $\varphi \in \mathscr{A}_{T}$. Indeed, this will imply that (2.1) holds in the usual sense away from $\Gamma_{T}$. Since further $u=0$ on $(G \times\{0\}) \cup \partial D_{T}$ in some weak sense, it follows by standard parabolic theory that $u=0$ on $(G \times\{0\}) \cup \partial D_{T}$ in the usual continuous sense. $k$ :

To prove (2.6) we multiply $\left(3.3_{k}\right)$ by $\varphi$, integrate over $G^{k}$, and sum over

$$
\begin{aligned}
0= & h \sum_{k=1}^{m} \int_{G^{k}}\left(u^{h}\right)_{t}^{-}(x, k h) \varphi(x, k h)-h \sum_{k=1}^{m} \int_{G^{k}} \Delta u^{h}(x, k h) \varphi(x, k h) \\
= & h \int_{G^{m}} \sum_{k=\lambda(x)}^{m}\left(u^{h}\right)_{t}^{-}(x, k h) \varphi(x, k h)+h \sum_{k=1}^{m} \int_{G^{k}} \nabla u^{h} \cdot \nabla \varphi \\
& -h \sum_{k=1}^{m} \int_{L}\left(\nabla u^{h} \cdot N_{0}^{k}\right) \varphi d s .
\end{aligned}
$$

Breaking the first integral on the right-hand side into $m$ integrals taken over the sets $\lambda(x)=j \quad(0 \leq j \leq m)$, we get

$$
\begin{aligned}
h \int_{G^{m}} & \sum_{k=\lambda(x)}^{m}\left(u^{h}\right)_{t}^{-} \varphi=h \sum_{j=1}^{m} \int_{G^{j} \backslash G^{j-1}} \sum_{k=j}^{m}\left(u^{h}\right)_{t}^{-} \varphi+h \int_{G^{0}} \sum_{k=1}^{m}\left(u^{h}\right)_{t}^{-} \varphi \\
= & \sum_{j=1}^{m} \int_{G^{j} \backslash G^{j-1}}\{[u(x, m h) \varphi(x, m h)-u(x,(j-1) h) \varphi(x,(j-1) h)] \\
& \left.-h \sum_{k=j-1}^{m-1} u(x, k h) \varphi_{t}(x, k h)\right\} d x \\
& -h \int_{G^{0}} \sum_{k=0}^{m-1} u(x, k h) \varphi_{t}(x, k h) d x \\
= & -\sum_{j=1}^{m} \int_{(j-1) h}^{j h} \int_{L} u(z,(j-1) h) \varphi(z,(j-1) h)\left|\frac{\partial z(s, t)}{\partial(s, t)}\right| d s d t \\
= & -h \sum_{k=1}^{m-1} \int_{G^{k+1}} u(x, k h) \varphi_{t}(x, k h) d x,
\end{aligned}
$$


since $\varphi(x, m h)=\varphi(x, T)=0, u(x, 0)=0$; here we used (3.13). Using the last computation in (3.15), we obtain

$$
\begin{aligned}
& \sum_{j=1}^{m} \int_{(j-1) h}^{j h} \int_{L} u(z,(j-1) h) \varphi(z,(j-1) h)\left|\frac{\partial z(s, t)}{\partial(s, t)}\right| d s d t \\
& \quad+h \sum_{k=1}^{m-1} \int_{G^{k+1}} u(x, k h) \varphi_{t}(x, k h)-h \sum_{k=1}^{m} \int_{G^{k}} \nabla u^{h} \cdot \nabla \varphi \\
& \quad+h \sum_{k=1}^{m} \int_{L}\left(\nabla u^{h} \cdot N_{0}^{k}\right) \varphi(z, k h) d s=0 .
\end{aligned}
$$

Since $\int_{0}^{T} \int_{L}\left|u^{h}\right| \leq C$, the first sum on the left-hand side of (3.16) is equal to

$$
\int_{0}^{T} \int_{L} u^{h}(z(s, t), t) \varphi(z(s, t), t)\left|\frac{\partial z(s, t)}{\partial(s, t)}\right|+O(h) .
$$

Next, for any smooth function $\eta$,

$$
\begin{aligned}
\int_{G_{T}} u^{h} \eta= & \sum_{j=1}^{m-1} \int_{j h}^{(j+1) h} d t \int_{G(t)} u^{h} \eta=h \sum_{j=1}^{m-1} \int_{G_{j+1}} u^{h}(x, j h) \eta(x, j h) \\
& -\sum_{j=1}^{m-1} \int_{j h}^{(j+1) h} d t \int_{t}^{(j+1) h} \int_{L} u^{h}(z, j h) \eta(z, j h)\left|\frac{\partial z(s, \tau)}{\partial(s, \tau)}\right| d s d \tau \\
& +\sum_{j=1}^{m-1} \int_{j h}^{(j+1) h} d t \int_{G(t)} u^{h}(x, j h)[\eta(x, t)-\eta(x, j h)] d x
\end{aligned}
$$

and the last two sums are $O(h)$ since $\int_{0}^{T} \int_{L}\left|u^{h}\right| \leq C$.

Next we obtain

$$
h \sum_{k=1}^{m} \int_{G^{k}} \nabla u^{h} \cdot \nabla \varphi=\int_{G_{T}} \nabla u^{h} \cdot \nabla \varphi+O(h)
$$

since $\int_{G_{T}}\left|\nabla u^{h}\right| \leq C$. Finally, to evaluate the last term on the left-hand side of (3.16) we use (3.4) and perform "integration by parts" in the $t$-variable. This leads to

$$
\begin{gathered}
\int_{0}^{T} \int_{L} u^{h}(z(s, t), t)\left\{\frac{d}{d t}(a \varphi)+\sum \frac{d}{d s_{j}}\left(b_{j} \varphi\right)-c \varphi\right\} \\
+\int_{0}^{T} \int_{L} \varphi f_{0} d s d t+O(h) .
\end{gathered}
$$

Taking $h \rightarrow 0$ in (3.16) and using the above estimates (with $\eta=\varphi_{t}$ ) we obtain the relation (2.6). This completes the proof of Theorem 3.1.

Remark 3.1. Notice that the $u_{h}$ 's satisfy

$$
\sup _{0<t<T}\left\{\int_{G(t)}\left(u^{h}\right)^{2}(x, t) d x+\int_{\Gamma(t)}\left(u^{h}\right)^{2}\left(x^{\prime}, t\right) d \sigma_{t}\left(x^{\prime}\right)\right\} \leq C_{T}
$$


with $C_{T}$ independent of $h$. Thus we may conclude that

$$
\sup _{0<t<T}\left\{\int_{G(t)} u^{2}(x, t)+\int_{\Gamma(t)} u^{2}\left(x^{\prime}, t\right) d \sigma_{t}\left(x^{\prime}\right)\right\} \leq C_{T} .
$$

Remark 3.2. Multiplying (2.1) by $u^{2 m-1}$ ( $m$ positive integer) and integrating over $G_{\tau}$ we can formally obtain an energy inequality analogous to (3.2), from which we deduce that

$$
\sup _{0<t<T}\left\{\int_{G(t)} u^{2 m}(x, t) d x+\int_{\Gamma(t)} u^{2 m}\left(x^{\prime}, t\right) d \sigma_{t}\left(x^{\prime}\right)\right\} \leq C_{T, m} .
$$

This can also be proved rigorously by finite differences.

\section{UNIQUENESS}

Theorem 4.1. The weak solution of (2.1)-(2.3) is unique.

Proof. Suppose $u_{1}, u_{2}$ are two solutions and let $u=u_{1}-u_{2}$. From (2.6) we deduce, for every $\eta \in \mathscr{A}_{T}$,

$$
\begin{aligned}
& -\int_{G_{T}} u \eta_{t}+\int_{G_{T}} \nabla u \cdot \nabla \eta \\
& \quad-\int_{0}^{T} \int_{L} u\left\{\frac{d}{d t}(a \eta)+\sum \frac{d}{d s_{j}}\left(b_{j} \eta\right)-c \eta+\left|\frac{\partial z}{\partial(s, t)}\right| \eta\right\} d s d t=0 .
\end{aligned}
$$

Let $\tau \in(0, T)$ and define

$$
\eta(x, t)=\left\{\begin{array}{l}
-\int_{\tau}^{t} u\left(x, t^{\prime}\right) d t^{\prime} \quad \text { if } t<\tau, \\
0 \quad \text { if } t>\tau .
\end{array}\right.
$$

Then $\eta=0$ on $\partial D_{T} \cup G(T)$ and

$$
\begin{gathered}
\nabla \eta(x, t)=\left\{\begin{array}{l}
-\int_{\tau}^{t} \nabla u\left(x, t^{\prime}\right) d t^{\prime} \quad \text { if } t<\tau, \\
0 \text { if } t>\tau,
\end{array}\right. \\
\eta_{t}= \begin{cases}-u & \text { if } t<\tau, \\
0 & \text { if } t>\tau .\end{cases}
\end{gathered}
$$

Notice that $\eta, \nabla \eta, \eta_{t}, \nabla \eta_{t} \in L^{2}\left(G_{T}\right)$ and therefore $\eta, \eta_{t}, \nabla \eta \in L^{2}\left(\Gamma_{T}\right)$. Now let $\varphi_{n}$ be a sequence of smooth functions satisfying:

$$
\begin{array}{ll}
\varphi_{n}=0 & \text { on } \partial D_{T}, \\
\varphi_{n}=0 & \text { for } t>\tau
\end{array}
$$

and

$$
\varphi_{n} \rightarrow \eta_{t} \quad \text { in } W
$$

where the norm in $W$ is given by (3.14) (in particular, $\varphi_{n} \rightarrow \eta_{t}$ in $L^{2}\left(\Gamma_{T}\right)$ ).

Then, if

$$
\Phi_{n}(x, t)=-\int_{T}^{t} \varphi_{n}\left(x, t^{\prime}\right) d t^{\prime}
$$


we have $\Phi_{n} \in \mathscr{A}_{T}$ so that, by (4.1),

$$
\begin{aligned}
& -\int_{G_{T}} u \Phi_{n t}+\int_{G_{T}} \nabla u \cdot \nabla \Phi_{n} \\
& \quad-\int_{0}^{T} \int_{L} u\left\{\frac{d}{d t}\left(a \Phi_{n}\right)+\sum \frac{d}{d s_{j}}\left(b_{j} \Phi_{n}\right)-c \Phi_{n}+\left|\frac{\partial z}{\partial(s, t)}\right| \Phi_{n}\right\}=0 .
\end{aligned}
$$

But since $\Phi_{n} \rightarrow \eta$ and $\Phi_{n t} \rightarrow \eta_{t}$ in $W$, if we let $n \rightarrow \infty$ in the above relation we conclude that $(4.1)$ holds also for $\eta$.

From (4.1) we get

$$
\int_{G_{\tau}} \eta_{t}^{2}-\int_{G_{\tau}} \nabla \eta \cdot \nabla \eta_{t}+\int_{0}^{\tau} \int_{L} \eta_{t}(z, t)\left\{a \frac{d \eta}{d t}+\sum b_{j} \frac{d \eta}{d s_{j}}+\gamma \eta\right\}=0
$$

where

$$
\gamma=\left|\frac{\partial z(s, t)}{\partial(s, t)}\right|+\frac{d a}{d t}+\sum \frac{d b_{j}}{d s_{j}}-c .
$$

We next wish to evaluate the expression

$$
\begin{aligned}
a \frac{d \eta}{d t} & +\sum_{j=1}^{n-1} b_{j} \frac{d \eta}{d s_{j}} \\
& =a \eta_{t}+h_{0}\left\{\left\|N_{0}\right\|^{2} \nabla \eta \cdot z_{t}+\sum_{j=1}^{n-1}\left(z_{t}+\nabla \theta_{0}\right) \cdot A_{j} N_{0}\left(\nabla \eta \cdot z_{s_{j}}\right)\right\} .
\end{aligned}
$$

Lemma 4.2.

$$
\begin{gathered}
\left(A_{j} N_{0}\right) \cdot N_{0}=0, \\
\left(A_{j} N_{0}\right) \cdot z_{s_{i}}=-\delta_{i j}\left\|N_{0}\right\|^{2} .
\end{gathered}
$$

Assuming the lemma for the moment, we claim that

$$
z_{t}=\frac{z_{t} \cdot N_{0}}{\left\|N_{0}\right\|^{2}} N_{0}-\frac{1}{\left\|N_{0}\right\|^{2}} \sum_{j=1}^{n-1}\left(z_{t} \cdot A_{j} N_{0}\right) z_{s_{j}}
$$

Indeed, for $z_{t}=N_{0}$ this follows from (4.7) whereas for $z_{t}=z_{s_{i}}$ this follows from (4.7) and (4.8). Since the vectors $N_{0}, z_{s_{1}}, \ldots, z_{s_{n-1}}$ span the entire space $\mathbf{R}^{n},(4.9)$ holds for any $z_{t}$.

From (4.7) it follows that $\nabla \theta_{0} \cdot A_{j} N_{0}=0$ (since $\nabla \theta_{0}$ is parallel to $N_{0}$ ). Therefore, from (4.6),

$$
\begin{aligned}
a \frac{d \eta}{d t} & +\sum b_{j} \frac{d \eta}{d s_{j}}=a \eta_{t}+h_{0}\left\|N_{0}\right\|^{2} \nabla \eta \cdot\left\{z_{t}+\sum_{j=1}^{n-1}\left(z_{t} \cdot A_{j} N_{0}\right) z_{s_{j}}\left\|N_{0}\right\|^{-2}\right\} \\
& =a \eta_{t}+h_{0}\left\|N_{0}\right\|^{2} \nabla \eta \cdot \frac{z_{t} \cdot N_{0}}{\left\|N_{0}\right\|^{2}} N_{0}=a \eta_{t}+\nabla \eta \cdot N_{0}
\end{aligned}
$$


since

$$
z_{t} \cdot N_{0}=-\nabla \theta_{0} \cdot N_{0}=\frac{1}{h_{0}} .
$$

The absence of tangential derivatives of $\eta$ on the right-hand side of $(4.10)$ is crucial for the proof of uniqueness.

Substituting (4.10) into (4.5) results in

$$
\int_{G_{\tau}} \eta_{t}^{2}-\int_{G_{\tau}} \nabla \eta \cdot \nabla \eta_{t}+\int_{0}^{\tau} \int_{L}\left(a \eta_{t}^{2}+\nabla \eta \cdot N_{0} \eta_{t}+\gamma \eta \eta_{t}\right)=0
$$

By integration by parts,

$$
\begin{aligned}
-\int_{G_{\tau}} \nabla \eta \cdot \nabla \eta_{t}= & -\frac{1}{2} \int_{G(\tau)}|\nabla \eta|^{2}+\frac{1}{2} \int_{G(0)}|\nabla \eta|^{2}(x, 0) d x \\
& +\frac{1}{2} \int_{G(\tau) \backslash G(0)}|\nabla \eta|^{2}(x, t(x)) d x
\end{aligned}
$$

and $\nabla \eta=0$ on $G(\tau)$. In the last integral we change variables $x=(z(s, t), t)$ to get

$$
\int_{0}^{\tau} \int_{L}|\nabla \eta|^{2}(z(s, t), t)\left(-\nabla \theta_{0} \cdot N_{0}\right) d s d t
$$

since

$$
\left|\frac{\partial z(s, t)}{\partial(s, t)}\right|=|\operatorname{det}| \begin{array}{cccc}
z_{1, t} & z_{1, s_{1}} & \cdots & z_{1, s_{n-1}} \\
z_{2, t} & z_{2, s_{1}} & \cdots & z_{2, s_{n-1}} \\
\vdots & \vdots & & \vdots \\
z_{n, t} & z_{n, s_{1}} & \cdots & z_{n, s_{n-1}}
\end{array} \mid=z_{t} \cdot N_{0}=-\nabla \theta_{0} \cdot N_{0} .
$$

It follows that

$$
\begin{aligned}
\int_{G_{\tau}} \eta_{t}^{2} & +\int_{0}^{\tau} \int_{L}\left[\frac{1}{2}|\nabla \eta|^{2}\left(-\nabla \theta_{0} \cdot N_{0}\right)+\frac{\left|N_{0}\right|^{2}}{\left(-\nabla \theta_{0} \cdot N_{0}\right)} \eta_{t}^{2}+\nabla \eta \cdot N_{0} \eta_{t}\right] \\
& \leq \int_{0}^{\tau} \int_{L}\left(\varepsilon \eta_{t}^{2}+\frac{C}{\varepsilon} \eta^{2}\right)
\end{aligned}
$$

for any $\varepsilon>0$.

By the Cauchy inequality, the expression in brackets in the integrand on the left-hand side of (4.11) is bigger than

$$
\frac{1}{6}|\nabla \eta|^{2}\left(-\nabla \theta_{0} \cdot N_{0}\right)+\frac{1}{8} \eta_{t}^{2} \frac{\left|N_{0}\right|^{2}}{\left(-\nabla \theta_{0} \cdot N_{0}\right)}
$$

Choosing $\varepsilon$ small we get

$$
\int_{G_{\tau}} \eta_{t}^{2}+\int_{0}^{\tau} \int_{L} \eta_{t}^{2} \leq C_{\varepsilon} \int_{0}^{\tau} \int_{L} \eta^{2}
$$

and since

$$
\eta(x, t)=\int_{\tau}^{t} \eta_{t}
$$


we deduce that $\eta=0$ if $\tau$ is small enough. This proves uniqueness for $t<\tau$. We can now proceed step-by-step to prove uniqueness for all $t<T$.

Proof of Lemma 4.2. We first consider a special case.

Lemma 4.3. Suppose that

$$
\begin{array}{cc}
\frac{\partial z_{i}}{\partial s_{j}}(s, t)=\delta_{i j}, & 1 \leq i, j \leq n-1, \\
\frac{\partial z_{n}}{\partial s_{j}}(s, t)=\gamma_{j}, & 1 \leq j \leq n-1 .
\end{array}
$$

Then

(a)

$$
\begin{aligned}
N_{1 i} & =\sum_{\substack{j=1 \\
j \neq i}}^{n-1}\left(\frac{\partial \zeta_{j}}{\partial s_{i}} \gamma_{j}-\frac{\partial \zeta_{j}}{\partial s_{j}} \gamma_{i}\right)-\frac{\partial \zeta_{n}}{\partial s_{i}}, \quad 1 \leq i \leq n-1, \\
N_{1 n} & =\sum_{j=1}^{n-1} \frac{\partial \zeta_{j}}{\partial s_{j}}
\end{aligned}
$$

(b)

$$
A_{j}=\left(a_{i k}^{j}\right)= \begin{cases}-\gamma_{i} & \text { if } k=j, 1 \leq i \leq n-1, i \neq j, \\ \gamma_{k} & \text { if } i=j, 1 \leq k \leq n-1, k \neq i, \\ -1 & \text { if } i=j, k=n, \\ 1 & \text { if } i=n, k=j, \\ 0 & \text { otherwise }\end{cases}
$$

for $1 \leq j \leq n-1$;

(c) For $1 \leq j \leq n-1$,

$$
A_{j} N_{0} \cdot N_{0}=0, \quad A_{j} N_{0} \cdot z_{s_{i}}=-\delta_{i j}\left\|N_{0}\right\|^{2} \quad(1 \leq i \leq n-1),
$$

where

$$
N_{0}=\left(\begin{array}{c}
-\gamma_{1} \\
-\gamma_{2} \\
\vdots \\
-\gamma_{n-1} \\
1
\end{array}\right) .
$$

Proof. Recall that $N_{1}$ was defined as

$$
N_{1 i}=\left.(-1)^{n+i} \frac{d}{d \varepsilon} \operatorname{det}\left(\frac{\partial\left(z_{1}+\varepsilon \zeta_{1}\right)}{\partial s}, \ldots,\left(\frac{\partial\left(z_{i}+\varepsilon \zeta_{i}\right)}{\partial s}\right)^{-}, \ldots, \frac{\partial\left(z_{n}+\varepsilon \zeta_{n}\right)}{\partial s}\right)\right|_{\varepsilon=0}
$$

where $1 \leq i \leq n$ and

$$
\left(\frac{\partial\left(z_{1}+\varepsilon \zeta_{1}\right)}{\partial s}, \ldots,\left(\frac{\partial\left(z_{i}+\varepsilon \zeta_{i}\right)}{\partial s}\right)^{\wedge}, \ldots, \frac{\partial\left(z_{n}+\varepsilon \zeta_{n}\right)}{\partial s}\right)
$$


denotes the matrix whose columns are

$$
\begin{gathered}
\left(\begin{array}{c}
\frac{\partial\left(z_{1}+\varepsilon \zeta_{1}\right)}{\partial s_{1}} \\
\vdots \\
\frac{\partial\left(z_{1}+\varepsilon \zeta_{1}\right)}{\partial s_{n-1}}
\end{array}\right), \ldots,\left(\begin{array}{c}
\frac{\partial\left(z_{i-1}+\varepsilon \zeta_{i-1}\right)}{\partial s_{1}} \\
\vdots \\
\frac{\partial\left(z_{i-1}+\varepsilon \zeta_{i-1}\right)}{\partial s_{n-1}}
\end{array}\right), \\
\left(\begin{array}{c}
\frac{\partial\left(z_{i+1}+\varepsilon \zeta_{i+1}\right)}{\partial s_{1}} \\
\vdots \\
\frac{\partial\left(z_{i+1}+\varepsilon \zeta_{i+1}\right)}{\partial s_{n-1}}
\end{array}\right), \ldots,\left(\begin{array}{c}
\frac{\partial\left(z_{n}+\varepsilon \zeta_{n}\right)}{\partial s_{1}} \\
\vdots \\
\frac{\partial\left(z_{n}+\varepsilon \zeta_{n}\right)}{\partial s_{n-1}}
\end{array}\right) .
\end{gathered}
$$

Thus

$$
N_{1 i}=(-1)^{n+i} \sum_{\substack{j=1 \\ j \neq i}}^{n} \operatorname{det}\left(\frac{\partial z_{1}}{\partial s}, \ldots,\left(\frac{\partial z_{i}}{\partial s}\right)^{-}, \ldots, \frac{\partial z_{j-1}}{\partial s}, \frac{\partial \zeta_{j}}{\partial s}, \frac{\partial z_{j+1}}{\partial s}, \ldots, \frac{\partial z_{n}}{\partial s}\right)
$$

Let

$B_{i j}=\left(\frac{\partial z_{1}}{\partial s}, \ldots,\left(\frac{\partial z_{i}}{\partial s}\right)^{\wedge}, \ldots, \frac{\partial z_{j-1}}{\partial s}, \frac{\partial \zeta_{j}}{\partial s}, \frac{\partial z_{j+1}}{\partial s}, \ldots, \frac{\partial z_{n}}{\partial s}\right) \quad(i \neq j)$.

Then, if $1 \leq i<j \leq n-1$,

$$
B_{i j}=\left(\begin{array}{ccccccccccccc}
1 & 0 & \cdots & 0 & 0 & 0 & \cdots & 0 & \frac{\partial \zeta_{j}}{\partial s_{1}} & 0 & \cdots & 0 & \gamma_{1} \\
0 & 1 & \cdots & 0 & 0 & 0 & \cdots & 0 & \frac{\partial \zeta_{j}}{\partial s_{2}} & 0 & \cdots & 0 & \gamma_{2} \\
\vdots & \vdots & \ddots & \vdots & \vdots & \vdots & \vdots & \vdots & \vdots & & \vdots & \vdots & \\
0 & 0 & \cdots & 1 & 0 & 0 & \cdots & 0 & \frac{\partial \zeta_{j}}{\partial s_{i-1}} & 0 & \cdots & 0 & \gamma_{i-1} \\
0 & 0 & \cdots & 0 & 0 & 0 & \cdots & 0 & \frac{\partial \zeta_{j}}{\partial s_{i}} & 0 & \cdots & 0 & \gamma_{i} \\
0 & 0 & \cdots & 0 & 1 & 0 & \cdots & 0 & \frac{\partial \zeta_{j}}{\partial s_{i+1}} & 0 & \cdots & 0 & \gamma_{i+1} \\
0 & 0 & \cdots & 0 & 0 & 1 & \cdots & 0 & \cdots & & \cdots & & \cdots \\
\vdots & \vdots & & & & & \ddots & \vdots & \vdots & \vdots & & \vdots & \vdots \\
0 & 0 & \cdots & & \cdots & & \cdots & 1 & \frac{\partial \zeta_{j}}{\partial s_{j-1}} & 0 & \cdots & 0 & \gamma_{j-1} \\
0 & 0 & \cdots & & \cdots & & \cdots & 0 & \frac{\partial \zeta_{j}}{\partial s_{j}} & 0 & \cdots & 0 & \gamma_{j} \\
0 & 0 & \cdots & & \cdots & & \cdots & 0 & \frac{\partial \zeta_{j}}{\partial s_{j+1}} & 1 & \cdots & 0 & \gamma_{j+1} \\
\vdots & \vdots & & & & & & \vdots & \vdots & \vdots & \ddots & \vdots & \vdots \\
0 & 0 & \cdots & & \cdots & & \cdots & 0 & \frac{\partial \dot{\zeta}_{j}}{\partial s_{n-1}} & 0 & \cdots & 1 & \gamma_{n-1}
\end{array}\right)
$$

that is

$$
B_{i j}=\left(\begin{array}{ccccc}
I_{i-1} & O_{(i-1) \times(j-i-1)} & \frac{\partial \zeta_{j}}{\partial\left(s_{1}, \ldots, s_{i-1}\right)} & O_{(i-1) \times(n-j-1)} & \Gamma_{1} \\
O_{1 \times(i-1)} & O_{1 \times(j-i-1)} & \frac{\partial \zeta_{j}}{\partial s_{i}} & O_{1 \times(n-j-1)} & \gamma_{i} \\
O_{(j-i-1) \times(i-1)} & I_{j-i-1} & \frac{\partial \zeta_{j}}{\partial\left(s_{i+1}, \ldots, s_{j-1}\right)} & O_{(j-i-1) \times(n-j-1)} & \Gamma_{2} \\
O_{1 \times(i-1)} & O_{1 \times(j-i-1)} & \frac{\partial \zeta_{j}}{\partial s_{j}} & O_{1 \times(n-j-1)} & \gamma_{j} \\
O_{(n-j-1) \times(i-1)} & O_{(n-j-1) \times(j-i-1)} & \frac{\partial \zeta_{j}}{\partial\left(s_{j+1}, \ldots, s_{n-1}\right)} & I_{n-j-1} & \Gamma_{3}
\end{array}\right)
$$


where

$$
\begin{gathered}
I_{k}=\text { identity matrix in } \mathbf{R}^{k \times k}, \\
O_{k \times l}=\text { zero matrix in } \mathbf{R}^{k \times l}, \\
\frac{\partial \zeta_{j}}{\partial\left(s_{k}, \ldots, s_{l}\right)}=\left(\begin{array}{c}
\frac{\partial \zeta_{j}}{\partial s_{k}} \\
\vdots \\
\frac{\partial \zeta_{j}}{\partial s_{l}}
\end{array}\right), \\
\Gamma_{1}=\left(\begin{array}{c}
\gamma_{1} \\
\gamma_{2} \\
\vdots \\
\gamma_{i-1}
\end{array}\right), \quad \Gamma_{2}=\left(\begin{array}{c}
\gamma_{i+1} \\
\gamma_{i+2} \\
\vdots \\
\gamma_{j-1}
\end{array}\right) \text { and } \Gamma_{3}=\left(\begin{array}{c}
\gamma_{j+1} \\
\vdots \\
\gamma_{n-1}
\end{array}\right) .
\end{gathered}
$$

Then, $\operatorname{det} B_{i j}=\operatorname{det} C_{i j}$ where

$$
C_{i j}=\left(\begin{array}{cccc}
O_{1 \times(j-i-1)} & \partial \zeta_{j} / \partial s_{i} & O_{1 \times(n-j-1)} & \gamma_{i} \\
I_{j-i-1} & \partial \zeta_{j} / \partial\left(s_{i+1}, \ldots, s_{j-1}\right) & O_{(j-i-1) \times(n-j-1)} & \Gamma_{2} \\
O_{1 \times(j-i-1)} & \partial \zeta_{j} / \partial s_{j} & O_{1 \times(n-j-1)} & \gamma_{j} \\
O_{(n-j-1) \times(j-i-1)} & \partial \zeta_{j} / \partial\left(s_{j+1}, \ldots, s_{n-1}\right) & I_{n-j-1} & \Gamma_{3}
\end{array}\right)
$$

and, by moving the column with $\partial \zeta_{j} / \partial s_{i}$ to the right,

$$
\operatorname{det} C_{i j}=(-1)^{n-j-1} \operatorname{det} D_{i j}
$$

where

$$
D_{i j}=\left(\begin{array}{cccc}
O_{1 \times(j-i-1)} & O_{1 \times(n-j-1)} & \partial \zeta_{j} / \partial s_{i} & \gamma_{i} \\
I_{j-i-1} & O_{(j-i-1) \times(n-j-1)} & \partial \zeta_{j} / \partial\left(s_{i+1}, \ldots, s_{j-1}\right) & \Gamma_{2} \\
O_{1 \times(j-i-1)} & O_{1 \times(n-j-1)} & \partial \zeta_{j} / \partial s_{j} & \gamma_{j} \\
O_{(n-j-1) \times(j-i-1)} & I_{n-j-1} & \partial \zeta_{j} / \partial\left(s_{j+1}, \ldots, s_{n-1}\right) & \Gamma_{3}
\end{array}\right) .
$$

After moving downward the rows with $\partial \zeta_{j} / \partial s_{i}, \partial \zeta_{j} / \partial s_{j}$, we get

$$
\operatorname{det} B_{i j}=(-1)^{n-j-1}(-1)^{n-i-2}(-1)^{n-j-1} \operatorname{det} E_{i j} \text {, }
$$

where

$$
E_{i j}=\left(\begin{array}{cccc}
I_{j-i-1} & O_{(j-i-1) \times(n-j-1)} & \partial \zeta_{j} / \partial\left(s_{i+1}, \ldots, s_{j-1}\right) & \Gamma_{2} \\
O_{(n-j-i) \times(j-i-1)} & I_{n-j-1} & \partial \zeta_{j} / \partial\left(s_{j+1}, \ldots, s_{n-1}\right) & \Gamma_{3} \\
O_{1 \times(j-i-1)} & O_{1 \times(n-j-1)} & \partial \zeta_{j} / \partial s_{i} & \gamma_{i} \\
O_{1 \times(j-i-1)} & O_{1 \times(n-j-1)} & \partial \zeta_{j} / \partial s_{j} & \gamma_{j}
\end{array}\right)
$$

and therefore

$$
\operatorname{det} B_{i j}=(-1)^{n-i}\left(\frac{\partial \zeta_{j}}{\partial s_{i}} \gamma_{j}-\frac{\partial \zeta_{j}}{\partial s_{j}} \gamma_{i}\right)
$$

On the other hand, if $1 \leq i<j=n$,

$$
B_{i j}=B_{i n}=\left(\begin{array}{ccc}
I_{i-1} & O_{i-1 \times(n-i-1)} & \partial \zeta_{n} / \partial\left(s_{1}, \ldots, s_{i-1}\right) \\
O_{1 \times(i-1)} & O_{1 \times(n-i-1)} & \partial \zeta_{n} / \partial s_{i} \\
O_{(n-i-1) \times(i-1)} & I_{n-i-1} & \partial \zeta_{n} / \partial\left(s_{i+1}, \ldots, s_{n-1}\right)
\end{array}\right)
$$


and, by moving the row with $\partial \zeta_{n} / \partial s_{i}$ to the bottom, $\operatorname{det} B_{i n}=(-1)^{n-i-1}$ $\partial \zeta_{n} / \partial s_{i}$

Next, if $1 \leq j<i=n$,

$$
B_{i j}=B_{n j}=\left(\begin{array}{ccc}
I_{j-1} & \partial \zeta_{j} / \partial\left(s_{1}, \ldots, s_{j-1}\right) & O_{(j-1) \times(n-j-1)} \\
O_{1 \times(j-1)} & \partial \zeta_{j} / \partial s_{j} & O_{1 \times(n-j-1)} \\
O_{(n-j-1) \times(j-1)} & \partial \zeta_{j} / \partial\left(s_{j+1}, \ldots, s_{n-1}\right) & I_{n-j-1}
\end{array}\right)
$$

and thus

$$
\operatorname{det} B_{n j}=\frac{\partial \zeta_{j}}{\partial s_{j}}
$$

Finally it is easy to check that formula (4.12) continues to be valid if $1 \leq$ $j<i \leq n-1$. Hence, if $1 \leq i \leq n-1$,

$$
\begin{aligned}
N_{1 i} & =(-1)^{n+i} \sum_{\substack{j=1 \\
j \neq i}}^{n} \operatorname{det} B_{i j} \\
& =(-1)^{n+i}\left\{\sum_{\substack{j=1 \\
j \neq i}}^{n-1}(-1)^{n-i}\left(\frac{\partial \zeta_{j}}{\partial s_{i}} \gamma_{j}-\frac{\partial \zeta_{j}}{\partial s_{j}} \gamma_{i}\right)+(-1)^{n-i-1} \frac{\partial \zeta_{n}}{\partial s_{i}}\right\} \\
& =\sum_{\substack{j=1 \\
j \neq i}}^{n-1}\left(\frac{\partial \zeta_{j}}{\partial s_{i}} \gamma_{i}-\frac{\partial \zeta_{j}}{\partial s_{j}} \gamma_{i}\right)-\frac{\partial \zeta_{n}}{\partial s_{i}}
\end{aligned}
$$

and

$$
N_{1 n}=\sum_{j=1}^{n-1} \operatorname{det} B_{n j}=\sum_{j=1}^{n-1} \frac{\partial \zeta_{j}}{\partial s_{j}}
$$

This completes the proof of (a). Next (b) is an immediate consequence of (a) and the relation $N_{1}=\sum_{j=1}^{n-1} A_{j} \partial \zeta / \partial s_{j}$ which defines the $A_{j}$.

Finally, let us prove (c). We have

$$
\begin{aligned}
&\left(A_{j} N_{0}\right)_{i}= \sum_{k=1}^{n} a_{i k}^{j} N_{0 k}=\sum_{k=1}^{n-1} a_{i k}^{j}\left(-\gamma_{k}\right)+a_{i n}^{j} \\
&=\gamma_{i} \gamma_{j}\left(1-\delta_{i n}\right)\left(1-\delta_{i j}\right)-\delta_{i j} \sum_{k=1}^{n-1} \gamma_{k}^{2}\left(1-\delta_{i k}\right)-\delta_{i j}-\delta_{i n} \gamma_{j} \\
& \quad(1 \leq j \leq n-1,1 \leq i \leq n) .
\end{aligned}
$$


Hence

$$
\begin{aligned}
& A_{j} N_{0} \cdot N_{0}=\sum_{i=1}^{n}\left(A_{j} N_{0}\right)_{i} \cdot N_{0 i} \\
& \quad=\sum_{i=1}^{n-1}\left(-\gamma_{i}\right)\left\{\gamma_{i} \gamma_{j}\left(1-\delta_{i n}\right)\left(1-\delta_{i j}\right)-\delta_{i j} \sum_{k=1}^{n-1} \gamma_{k}^{2}\left(1-\delta_{i k}\right)-\delta_{i j}-\delta_{i n} \gamma_{j}\right\}+\left(-\gamma_{j}\right) \\
& =\left(-\gamma_{j}\right) \sum_{i=1}^{n-1} \gamma_{i}^{2}\left(1-\delta_{i j}\right)+\gamma_{j} \sum_{k=1}^{n-1} \gamma_{k}^{2}\left(1-\delta_{j k}\right)+\gamma_{j}-\gamma_{j}=0
\end{aligned}
$$

and, for $1 \leq i \leq n-1$,

$$
\begin{aligned}
A_{j} N_{0} & \cdot z_{s_{i}}=\sum_{k=1}^{n}\left(A_{j} N_{0}\right)_{k} \cdot \frac{\partial z_{k}}{\partial s_{i}} \\
& =\left(A_{j} N_{0}\right)_{i}+\left(A_{j} N_{0}\right)_{n} \gamma_{i} \\
& =\left\{\gamma_{i} \gamma_{j}\left(1-\delta_{i j}\right)-\delta_{i j} \sum_{k=1}^{n-1} \gamma_{k}^{2}\left(1-\delta_{i k}\right)-\delta_{i j}\right\}+\gamma_{i}\left(-\gamma_{j}\right) \\
& =-\delta_{i j}\left\{\gamma_{i} \gamma_{j}+\sum_{k=1}^{n-1} \gamma_{k}^{2}\left(1-\delta_{i k}\right)+1\right\} \\
& =-\delta_{i j}\left(1+\sum_{k=1}^{n-1} \gamma_{k}^{2}\right)=-\delta_{i j}\left\|N_{0}\right\|^{2} .
\end{aligned}
$$

Having completed the proof of Lemma 4.3 we now proceed to prove Lemma 4.2.

Choose a local coordinate system $\tau=\tau(s)$ such that $z=z(\tau)$ satisfies the assumptions in Lemma 4.3. Then, by Lemma 4.3, the corresponding $N_{0}=$ $N_{0}^{\tau}, N_{1}=N_{1}^{\tau}$ and $A_{j}=A_{j}^{\tau}$ satisfy:

$$
\begin{gathered}
A_{j}^{\tau} N_{0}^{\tau} \cdot N_{0}^{\tau}=0, \\
A_{j} N_{0}^{\tau} \cdot z_{\tau_{j}}=-\delta_{i_{j}}\left\|N_{0}^{\tau}\right\|^{2} .
\end{gathered}
$$

Since clearly

$$
\left(\frac{\partial(z+\varepsilon \zeta)}{\partial \tau}\right)\left(\frac{\partial \tau}{\partial s}\right)=\left(\frac{\partial(z+\varepsilon \zeta)}{\partial s}\right),
$$

we deduce by taking determinants of both sides, differentiating in $\varepsilon$ and setting $\varepsilon=0$,

and, of course, also

$$
N_{1}^{s}=\delta N_{1}^{\tau}
$$

$$
N_{0}^{s}=\delta N_{0}^{\tau}
$$

where $\delta=\operatorname{det}(\partial \tau / \partial s)$; here $N_{0}^{s}$ and $N_{1}^{s}$ denote $N_{0}$ and $N_{1}$ in the $s$-coordinates. Since

$$
\frac{\partial \zeta}{\partial \tau_{j}}=\sum_{\lambda} \frac{\partial \zeta}{\partial s_{\lambda}} \frac{\partial s_{\lambda}}{\partial \tau_{j}}
$$


we find that

$$
\sum_{\lambda} A_{\lambda}^{s} \frac{\partial \zeta}{\partial s_{\lambda}}=N_{1}^{s}=\delta N_{1}^{\tau}=\delta \sum_{j} A_{j}^{\tau} \frac{\partial \zeta}{\partial \tau_{j}}=\delta \sum_{\lambda, j} A_{j}^{\tau} \frac{\partial \zeta}{\partial s_{\lambda}} \frac{\partial s_{\lambda}}{\partial \tau_{j}}
$$

It follows that

$$
A_{\lambda}^{s}=\delta \sum_{j=1}^{n-1} A_{j}^{\tau} \frac{\partial s_{\lambda}}{\partial \tau_{j}}
$$

Consequently

$$
\begin{aligned}
\left(A_{\lambda}^{s} N_{0}^{s}\right) \cdot N_{0}^{s} & =\delta^{2} A_{\lambda}^{s} N_{0}^{\tau} \cdot N_{0}^{\tau}=\delta^{3}\left(\sum A_{j}^{\tau} \frac{\partial s_{\lambda}}{\partial \tau_{j}} N_{0}^{\tau}\right) \cdot N_{0}^{\tau} \\
& =\delta^{3} \sum \frac{\partial s_{\lambda}}{\partial \tau_{j}}\left(A_{j}^{\tau} N_{0}^{\tau}\right) \cdot N_{0}^{\tau}=0
\end{aligned}
$$

by (4.13). Also

$$
\begin{aligned}
A_{\lambda}^{s} N_{0}^{s} \cdot z_{s_{i}} & =\delta^{2} \sum \frac{\partial s_{\lambda}}{\partial \tau_{j}}\left(A_{j}^{\tau} N_{0}^{\tau}\right) \cdot z_{s_{i}} \\
& =\delta^{2} \sum_{j, \mu} \frac{\partial s_{\lambda}}{\partial \tau_{j}}\left(A_{j}^{\tau} N_{0}^{\tau} \cdot z_{\tau_{\mu}}\right) \frac{\partial \tau_{\mu}}{\partial s_{i}} \\
& =-\delta^{2} \sum_{j, \mu} \frac{\partial s_{\lambda}}{\partial \tau_{j}}\left\|N_{0}^{\tau}\right\|^{2} \delta_{j \mu} \frac{\partial \tau_{\mu}}{\partial s_{i}} \quad(\text { by }(4.14)) \\
& =-\delta^{2} \sum_{j} \frac{\partial s_{\lambda}}{\partial \tau_{j}} \frac{\partial \tau_{j}}{\partial s_{i}}\left\|N_{0}^{\tau}\right\|^{2}=-\delta_{\lambda i} \delta^{2}\left\|N_{0}^{\tau}\right\|^{2}=-\delta_{\lambda i}\left\|N_{0}^{s}\right\|^{2}
\end{aligned}
$$

and Lemma 4.2 follows.

We conclude this section with several remarks.

Remark 4.1. In the definition of weak solution $u$ we used a specific parametrization $s$ of the smooth free boundary, namely $x=z(s, t)$. If we use another coordinate system $\tau$, then we get another weak solution $u=u^{\tau}$. Using the transformation (4.15) it is easy to verify that if $u$ is a weak solution with respect to the parametrization $s$ then it is also a weak solution with respect to the parametrization $\tau$.

Remark 4.2. If $u$ is a weak solution in $G_{T}$ then, as can easily be verified, it is also a weak solution in $G_{T^{\prime}}$ for any $T^{\prime}<T$. This remark together with Theorem 4.1 allows us to construct $u$ for all $t>0$ provided $\left(\theta_{0}, z\right)$ is smooth for all $t>0$. In $\S \S 6,7$ we shall take $\left(\theta_{0}, z\right)$ smooth for all $t>0$ and study the free boundary of $\theta_{0}+\varepsilon u$, i.e., the surface

$$
x=z(s, t)+\varepsilon \zeta(s, t), \quad \text { for } 0<t<\infty .
$$


Remark 4.3. Uniqueness for smooth solutions of (2.1)-(2.3) can be established by the maximum principle, as in [4].

\section{Auxiliary RESUlts IN ODE}

In the next two sections we shall be working with solutions to the ODE problem

$$
\begin{gathered}
\mathscr{L}_{n} w \equiv-w^{\prime \prime}-\left(\frac{n-3}{y}+\frac{y}{2}\right) w^{\prime}+\frac{n-3}{y^{2}} w=0, \quad 0<y<1, \\
\frac{1}{2} w^{\prime}(1)+\frac{2 n-3}{4} w(1)=\frac{2 n-1}{4}, \\
w(0+)=0 .
\end{gathered}
$$

In this section we establish existence and properties of the solution to (5.1)(5.3).

Theorem 5.1. If $n \geq 3$ there exists a unique solution $w$ of (5.1)-(5.3); further

$$
\begin{gathered}
w(y)>0, \quad w^{\prime}(y)>0 \text { if } 0<y<1, \\
w(1)>1 .
\end{gathered}
$$

Theorem 5.2. If $n=2$ there exists a solution $w$ of (5.1)-(5.3) satisfying (5.4), (5.5); $w$ is the (unique) minimal nonnegative solution of (5.1)-(5.3).

Proof of Theorem 5.1. First notice that, if $n=3,(5.1)-(5.3)$ becomes

$$
\begin{gathered}
-w^{\prime \prime}-\frac{y}{2} w^{\prime}=0, \quad 0<y<1, \\
\frac{1}{2} w^{\prime}(1)+\frac{3}{4} w(1)=\frac{5}{4}, \\
w(0+)=0
\end{gathered}
$$

and this problem can be solved explicitly. Indeed the unique solution $w$ is given by

$$
w(y)=\frac{5}{2+3 \alpha_{0}} \int_{0}^{y} e^{\left(1-z^{2}\right) / 4} d z
$$

where $\alpha_{0}=\int_{0}^{1} e^{\left(1-z^{2}\right) / 4} d z>1$. In particular, $w$ satisfies (5.4) and (5.5).

Thus we may assume $n \geq 4$. For any $\varepsilon>0$ consider the problem$$
\left(P_{\varepsilon}^{\infty}\right) \quad w(\varepsilon)=0,
$$$$
\mathscr{L}_{n} w=0, \quad \varepsilon<y<1,
$$$$
\frac{1}{2} w^{\prime}(1)+\frac{2 n-3}{4} w(1)=\frac{2 n-1}{4} \text {. }
$$

Since $n \geq 4$ the coefficient of $w$ in $\mathscr{L}_{n} w$ is $>0$; hence there exists a unique solution $w_{\varepsilon}$; furthermore, the maximum principle can be applied. We deduce that $w_{\varepsilon}(y)>0$ if $\varepsilon<y \leq 1$ and

$$
w_{\varepsilon}^{\prime}(\varepsilon)>0 .
$$


We compute that

$$
\mathscr{L}_{n} w_{\varepsilon}^{\prime}+\left(\frac{n-3}{y^{2}}-\frac{1}{2}\right) w_{\varepsilon}^{\prime}=\left(\mathscr{L}_{n} w_{\varepsilon}\right)^{\prime}+\frac{2(n-3)}{y^{3}} w_{\varepsilon} \geq 0
$$

and therefore $w_{\varepsilon}^{\prime}$ cannot take nonpositive minimum in $\varepsilon<y<1$. If $w_{\varepsilon}^{\prime}$ takes nonpositive minimum at $y=1$ then $w_{\varepsilon}^{\prime \prime}(1) \leq 0, w_{\varepsilon}^{\prime}(1) \leq 0$, and (since $\left.w_{\varepsilon}(1)>0\right)$ we get $\mathscr{L}_{n} w_{\varepsilon}(1)>0$, a contradiction. Recalling finally (5.6) we deduce that

$$
w_{\varepsilon}^{\prime}(y)>0 \text { if } \varepsilon \leq y \leq 1 .
$$

By applying the maximum principle to $w_{\varepsilon}$ we also deduce that $w_{\varepsilon}(y) \leq$ $w_{\varepsilon}(1)$, and by (5.2), (5.7),

$$
w_{\varepsilon}(y) \leq \frac{2 n-1}{2 n-3}, \quad \varepsilon \leq y \leq 1
$$

by comparison we also have

$$
w_{\varepsilon^{\prime}}(y) \leq w_{\varepsilon}(y) \text { if } \varepsilon<\varepsilon^{\prime} .
$$

It follows that $w(y) \equiv \lim _{\varepsilon \rightarrow 0} w_{\varepsilon}(y)$ exists and satisfies $\mathscr{L}_{n} w=0$ and the asserted boundary condition (5.2) at $y=1$, and $w^{\prime}(y)>0$ if $0<y \leq 1$.

Next, for any $0<\lambda<1, \mathscr{L}_{n} y^{\lambda}>0$ if $0<y<y_{0}$ for some $y_{0} \in(0,1)$. Hence,

$$
w_{\varepsilon}(y) \leq C y^{\lambda} \quad \text { if } \varepsilon<y<y_{0} \quad\left(C=\frac{2 n-1}{2 n-3} y_{0}^{-\lambda}\right),
$$

where (5.8) was used. We conclude that

$$
w(y) \leq C y^{\lambda}, \quad 0<y<y_{0},
$$

and, in particular, $w(0)=0$.

We have proved that $w$ satisfies (5.1)-(5.3). By the maximum principle, the solution to (5.1)-(5.3) is unique.

Observe next that the function $z(y) \equiv y$ satisfies the boundary condition (5.2) and $\mathscr{L}_{n} z<0$. Hence, by comparison, $w(y)>z(y)$ if $0<y \leq 1$ and, in particular, $w(1)>1$.

Proof of Theorem 5.2. Equations (5.1), (5.2) become

$$
\begin{gathered}
\mathscr{L}_{2} w \equiv-w^{\prime \prime}+\left(\frac{1}{y}-\frac{y}{2}\right) w^{\prime}-\frac{w}{y^{2}}=0, \\
\frac{1}{2} w^{\prime}(1)+\frac{1}{4} w(1)=\frac{3}{4} .
\end{gathered}
$$

Set

$$
\mathscr{L}_{0} w=-w^{\prime \prime}+\left(\frac{1}{y}-\frac{y}{2}\right) w^{\prime} .
$$

For any $\varepsilon<1, \varepsilon$ near 1 , by working with

$$
\tilde{w}_{\varepsilon}(y)=\frac{w_{\varepsilon}((1-\varepsilon) y+\varepsilon)}{1-\varepsilon} \quad(0<y<1)
$$


which satisfies: $\tilde{w}_{\varepsilon}^{\prime \prime}=O(1-\varepsilon), \tilde{w}_{\varepsilon}(0)=0, \tilde{w}_{\varepsilon}^{\prime}(1) \approx \frac{3}{2}$, we find that there exists a unique solution $w_{\varepsilon}(y)$ of $\left(P_{\varepsilon}^{\infty}\right)$ (with $\left.n=2\right)$. We denote by $\varepsilon_{0}$ the infimum of $\varepsilon$ such that for any $\varepsilon \leq \varepsilon^{\prime}<1$ there exists a unique positive solution $w_{\varepsilon^{\prime}}$ of $\left(P_{\varepsilon^{\prime}}^{\infty}\right) \quad(n=2)$. We first establish some properties of $w_{\varepsilon}$.

Integrating (5.11) in $y, \varepsilon<y<1$, and using

$$
\int_{\varepsilon}^{1}\left(\frac{1}{y}-\frac{y}{2}\right) w^{\prime}=\left[\frac{w}{y}-\frac{y w}{2}\right]_{\varepsilon}^{1}+\int_{\varepsilon}^{1}\left(\frac{w}{y^{2}}+\frac{w}{2}\right)
$$

and (5.12), we get

$$
w_{\varepsilon}(1)+\frac{1}{2} \int_{\varepsilon}^{1} w_{\varepsilon}+w_{\varepsilon}^{\prime}(\varepsilon)=\frac{3}{2}, \quad w_{\varepsilon}^{\prime}(\varepsilon) \geq 0,
$$

and, in particular,

$$
w_{\varepsilon}(1)<\frac{3}{2}
$$

Since

$$
\mathscr{L}_{0} w_{\varepsilon}=\frac{w_{\varepsilon}}{y^{2}} \geq 0,
$$

$w_{\varepsilon}$ cannot take a minimum in $\varepsilon<y \leq 1$ (we use here the boundary condition (5.12) and (5.15)) and, further,

$$
w_{\varepsilon}^{\prime}(\varepsilon)>0 .
$$

It follows that $w_{\varepsilon}(y)$ is either monotone increasing in $y$ or else first increases and then decreases. The latter possibility leads to $w_{\varepsilon}^{\prime}(1) \leq 0$ and then, by (5.12), $w_{\varepsilon}(1)>3$ which is a contradiction to $(5.15)$. Consequently,

$$
w_{\varepsilon}^{\prime}(y) \geq 0 \text { for } \varepsilon<y<1 .
$$

Next we show that

$$
w_{\varepsilon}(y)>w_{\varepsilon^{\prime}}(y) \text { if } \varepsilon^{\prime} \leq y \leq 1, \varepsilon<\varepsilon^{\prime} .
$$

Indeed, $w_{\varepsilon}(y)>\lambda w_{\varepsilon^{\prime}}(y)$ for $\varepsilon^{\prime} \leq y \leq 1$ if $\lambda$ is positive and small. Denoting by $\lambda_{0}$ the supremum of all $\lambda$ 's for which the inequality holds, it suffices to show that $\lambda_{0}>1$. Suppose $\lambda_{0} \leq 1$ and consider $\zeta=w_{\varepsilon}-\lambda_{0} w_{\varepsilon^{\prime}}$. Then

$$
\begin{gathered}
\mathscr{L}_{0} \zeta=\frac{\zeta}{y^{2}} \geq 0, \quad \varepsilon^{\prime} \leq y \leq 1, \\
\zeta\left(\varepsilon^{\prime}\right)>0, \quad \frac{1}{2} \zeta^{\prime}(1)+\frac{1}{4} \zeta(1) \geq 0 .
\end{gathered}
$$

It follows that $\zeta(y)>0$ if $\varepsilon^{\prime} \leq y \leq 1$, and thus $\zeta \geq \delta w_{\varepsilon^{\prime}}$ for some $\delta>0$, a contradiction to the definition of $\lambda_{0}$.

By (5.15), (5.17), (5.18), we see that if $\varepsilon_{0}>0$ then $w_{0} \equiv \lim _{\varepsilon \rightarrow \varepsilon_{0}} w_{\varepsilon}$ exists and is a solution of $\left(P_{\varepsilon_{0}}^{\infty}\right)$, satisfying (5.17). We shall derive a contradiction, thereby proving that $\varepsilon_{0}$ must be equal to zero.

Let $w$ be the solution of (5.11) under the boundary conditions (5.12) and $w(1)=w_{0}(1)+\delta$, i.e., $w(1)=w_{0}(1)+\delta$ and $w^{\prime}(1)=\frac{3}{2}+\frac{1}{2} w_{0}(1)-\frac{1}{2} \delta$ for 
$\delta>0, \delta$ small. Then, by continuity, (using the fact $w_{0}^{\prime}\left(\varepsilon_{0}\right)>0$ ) $w(y)>0$ for $\varepsilon<y<1, w(\varepsilon)=0$, for some $\varepsilon$ near $\varepsilon_{0}$. If $\varepsilon>\varepsilon_{0}$ then $w=w_{\varepsilon}$ and we get a contradiction to (5.18) at $y=1$. Hence $\varepsilon<\varepsilon_{0}$ and, by varying $\delta$ we get positive solutions $w_{\varepsilon}(y)$ of $\left(P_{\varepsilon}^{\infty}\right)$ for all $\varepsilon_{1} \leq \varepsilon \leq \varepsilon_{0}$, for some $0<\varepsilon_{1}<\varepsilon_{0}$. We claim that $w_{\varepsilon}$ is the unique nonnegative solution of $\left(P_{\varepsilon}^{\infty}\right)$. Indeed, if $\hat{w}_{\varepsilon}$ is another nonnegative solution then we can proceed as in the proof of (5.18) (noting that $w_{\varepsilon}-\lambda \hat{w}_{\varepsilon} \geq 0$ implies $\left.w_{\varepsilon}^{\prime}(0)-\lambda w_{\varepsilon}^{\prime}(0)>0\right)$ to show that $w_{\varepsilon} \geq \lambda \hat{w}_{\varepsilon}$ for all $0 \leq \lambda \leq 1$, so that $w_{\varepsilon} \geq \hat{w}_{\varepsilon}$, and similarly $\hat{w}_{\varepsilon} \geq w_{\varepsilon}$.

Having proved that for each $\varepsilon_{1}<\varepsilon \leq \varepsilon_{0}$ there is a unique nonnegative solution of $\left(P_{\varepsilon}^{\infty}\right)$, we get a contradiction to the definition of $\varepsilon_{0}$. It follows that $\varepsilon_{0}=0$.

From (5.15), (5.17), (5.18) and the fact that $\varepsilon_{0}=0$ it follows that

$$
w(y)=\lim _{\varepsilon \rightarrow 0} w_{\varepsilon}(y)
$$

exists, and it satisfies (5.1), (5.2), (5.4). (If $w^{\prime}(0)=0,0<y_{0}<1$, then $w^{\prime \prime}\left(y_{0}\right)<0$, by $(5.1)$, so that $w^{\prime}(y)<0$ for $y>y_{0}, y-y_{0}$ small, which is a contradiction.)

To prove (5.3) we integrate (5.11) over $\varepsilon<y<1$ and use (5.12), and (5.13); we get

$$
w(1)+\frac{1}{2} \int_{\varepsilon}^{1} w+\left[w^{\prime}(\varepsilon)-\frac{w(\varepsilon)}{\varepsilon}\right]+\frac{1}{2} \varepsilon w(\varepsilon)=\frac{3}{2} .
$$

Taking $\varepsilon \rightarrow 0$ in (5.14) and comparing with (5.19), we deduce that

$$
\varliminf_{\varepsilon \rightarrow 0}\left(w^{\prime}(\varepsilon)-\frac{w(\varepsilon)}{\varepsilon}\right) \geq 0 .
$$

If $w(0+)>0$, this leads to $w^{\prime}(\varepsilon)>c / \varepsilon \quad(c>0)$ as $\varepsilon \rightarrow 0$, a contradiction. Hence $w(0)=0$.

To prove that $w(1)>1$ notice that if $w(1) \leq 1$ then, from (5.19) it follows that

$$
w^{\prime}(\varepsilon)-\frac{w(\varepsilon)}{\varepsilon} \geq c>0, \quad 0<\varepsilon<\varepsilon_{1},
$$

for some constants $c, \varepsilon_{1}$. Using this in (5.11) we get

$$
w^{\prime \prime}(y)+\frac{y}{2} w^{\prime}(y) \geq \frac{c}{y}, \quad 0<y<\varepsilon_{1},
$$

or

$$
\left(w^{\prime} e^{y^{2} / 4}\right)^{\prime} \geq \frac{c}{y}
$$

Hence, since $w^{\prime} \geq 0$,

$$
w^{\prime}(y) e^{y^{2} / 4} \geq \int_{\varepsilon}^{y} \frac{c}{z} d z=c \log \frac{y}{\varepsilon} \rightarrow \infty \quad \text { if } \varepsilon \rightarrow 0,
$$

a contradiction.

We next prove that $w(y)$ is the (unique) minimal nonnegative solution of (5.1)-(5.3). Let $\tilde{w}$ be any nonnegative solution of (5.1)-(5.3). Since $\mathscr{L}_{0} \tilde{w} \geq 0$, 
$\tilde{w}(y)>0$ if $0<y<1$. For any $\varepsilon>0, \tilde{w} \geq \lambda w_{\varepsilon}$ for $\varepsilon \leq y \leq 1$ if $\lambda$ is positive and sufficiently small. As before we can argue that the supremum of such $\lambda$ 's must be $\geq 1$, so that $\tilde{w} \geq w_{\varepsilon}$. Taking $\varepsilon \rightarrow 0$ we see that $\tilde{w} \geq w$, i.e., $w$ is the minimal solution.

\section{THE EFFECT OF SURFACE TENSION ON THE INTERFACE (RADIAL CASE)}

If $G$ is a shell $\left\{r_{0}<|x|<r_{1}\right\}$ and the data $\tilde{\theta}$ are radially symmetric, then we can try to solve the Stefan problem with surface tension by a function $\theta(r, t)$ where $r=|x|$. The free boundary conditions are

$$
\theta=\frac{\varepsilon}{s_{\varepsilon}(t)}, \quad-\theta_{r}=\frac{d s_{\varepsilon}(t)}{d t} \quad \text { on } r=s_{\varepsilon}(t) .
$$

The method of integral equations used in case $\varepsilon=0$ (e.g. [5]) can be used also for $\varepsilon>0$ to show that for any large $T$ there is a small $\varepsilon_{0}=\varepsilon(T)$ positive such that a solution exists for $0 \leq t \leq T$ if $0<\varepsilon<\varepsilon_{0}$; furthermore, the solution depends smoothly on $\varepsilon$.

In this section we consider a special radial solution with free boundary $s(t)=$ $\sqrt{t}$ when $\varepsilon=0$ and investigate the effect of the $\varepsilon$-approximating $u$ on the free boundary. Our interest is not really in this special radial case, but rather in the method which will be developed to tackle it, for this method will be used in the next section to study the general (nonradial) case. We consider $\theta_{0}(x, t)$, a function of $|x|$ and $t$,

$$
\theta_{0}(x, t)=f\left(\frac{|x|}{\sqrt{t}}\right), \quad t>1,
$$

and the free boundary

$$
|x|=\sqrt{t}, \quad t>1 .
$$

One easily finds (see $[6$, p. 87]) that

$$
\begin{gathered}
f(z)=c_{0} \int_{z}^{\infty} \zeta^{1-n} e^{-\zeta^{2} / 4} d \zeta-c_{1}, \\
c_{0}=\frac{1}{2} e^{1 / 4}, \quad c_{1}=\frac{1}{2} e^{1 / 4} \int_{1}^{\infty} \zeta^{1-n} e^{-\zeta^{2} / 4} d \zeta .
\end{gathered}
$$

We take

$$
D=\{|x|<\alpha\} \text { for some } 0<\alpha<1
$$

and this determines the initial and boundary values $\tilde{\theta}$ of $\theta_{0}$ on $G \times\{1\} \quad(G=$ $\{\alpha<|x|<1\})$ and on $\partial D_{\infty}=\{|x|=\alpha, 1<t<\infty\}$. Notice that

$$
\tilde{\theta} \rightarrow \frac{1}{2} e^{1 / 4} \int_{0}^{1} e^{-\zeta^{2} / 4} \zeta^{n-1} d \zeta \quad \text { as } t \rightarrow \infty \text { on } \partial D_{\infty} .
$$

The free boundary can be represented in the form

$$
x=z(s, t)=\sqrt{t} \nu_{0}, \quad \nu_{0}=\frac{x}{|x|} .
$$


Clearly (1.22), (1.23) remain true if we replace $N_{0}$ by $\gamma(s, t) N_{0}$ and the $A_{j}$ 's and $h_{0}$ accordingly (i.e., $A_{j}$ by $\gamma A_{j}$ and $h_{0}$ by $\left.h_{0} / \gamma\right)$. Thus, we may take

$$
N_{0}=z(s, t)=\sqrt{t} \nu_{0} \text {. }
$$

Since

$$
\begin{aligned}
& f^{\prime}(z)=-\frac{1}{2} e^{1 / 4} z^{1-n} e^{-z^{2} / 4}, \\
& f^{\prime \prime}(z)=\frac{1}{2} e^{1 / 4}\left(\frac{1}{2} z^{2-n}+(n-1) z^{-n}\right) e^{-z^{2} / 4}
\end{aligned}
$$

we have

$$
f^{\prime}(1)=-\frac{1}{2}, \quad f^{\prime \prime}(1)=\frac{2 n-1}{4} .
$$

Next, on the free boundary,

$$
z_{t}+\nabla \theta_{0}=\frac{1}{2 \sqrt{t}} \nu_{0}+f^{\prime}(1) \frac{x}{|x| \sqrt{t}}=0
$$

by (6.4); also

$$
\nabla \theta_{0} \cdot N_{0}=f^{\prime}(1) \frac{x}{|x| \sqrt{t}} \cdot \sqrt{t} \nu_{0}=f^{\prime}(1)=-\frac{1}{2}
$$

so that

$$
h_{0}=-\frac{1}{\nabla \theta_{0} \cdot N_{0}}=2
$$

Thus

$$
a=N_{0} \cdot N_{0} h_{0}=2 t
$$

Next

$$
\begin{aligned}
\nabla \partial_{x_{i}} \theta_{0} & =\partial_{x_{i}} \nabla \theta_{0}=\partial_{x_{i}} f^{\prime}\left(\frac{|x|}{\sqrt{t}}\right) \frac{x}{|x| \sqrt{t}} \\
& =f^{\prime \prime}\left(\frac{|x|}{\sqrt{t}}\right) \frac{x_{i}}{|x| \sqrt{t}} \frac{x}{|x| \sqrt{t}}+f^{\prime}\left(\frac{|x|}{\sqrt{t}}\right) \frac{1}{\sqrt{t}}\left(\frac{e_{i}}{|x|}-\frac{x x_{i}}{|x|^{3}}\right)
\end{aligned}
$$

where $e_{i}$ is the vector with components $\delta_{i j}$. Hence, on $|x|=\sqrt{t}$,

$$
\nabla \partial_{x_{i}} \theta_{0} \cdot N_{0}=f^{\prime \prime}(1) \frac{x_{i}}{|x| \sqrt{t}}+f^{\prime}(1)\left(\frac{x_{i}}{|x|^{2}}-\frac{x_{i}}{|x|^{2}}\right)=\frac{2 n-1}{4} \frac{x_{i}}{|x| \sqrt{t}}
$$

by (6.4), and

$$
\begin{aligned}
N_{0} \cdot( & \left.\nabla \partial_{x_{1}} \theta_{0} \cdot N_{0}, \ldots, \nabla \partial_{x_{n}} \theta_{0} \cdot N_{0}\right) \\
& =\frac{2 n-1}{4} \frac{1}{|x| \sqrt{t}} x \cdot \sqrt{t} \frac{x}{|x|}=\frac{2 n-1}{4} .
\end{aligned}
$$

From the definition of $b_{j}$ in (1.23) and (6.5) we see that

$$
b_{j}=0 \text {. }
$$


From the definition of $c$ in (1.23) and (6.5), (6.6), (6.8) we get

$$
c=h_{0}\left\{N_{0} \cdot N_{0 t}+\frac{2 n-1}{4}\right\}=2\left\{\sqrt{t} \frac{x}{|x|} \cdot \frac{1}{2 \sqrt{t}} \frac{x}{|x|}+\frac{2 n-1}{4}\right\},
$$

or,

$$
c=\frac{2 n+1}{2}
$$

Next

$$
N_{0} \cdot \nabla u=\sqrt{t} \frac{x}{|x|} \cdot \nabla u=\sqrt{t} u_{r}
$$

where $r=|x|$. Finally, since the mean curvature of $|x|=\sqrt{t}$ is $\kappa=1 / \sqrt{t}$,

$$
\begin{aligned}
f_{0} & =t \frac{d}{d t}(2 \kappa)+\left\{N_{0} \cdot N_{0 t}+\frac{2 n-1}{4}\right\} 2 \kappa \\
& =2 t\left(-\frac{1}{2}\right) t^{-3 / 2}+\frac{2 n+1}{2 \sqrt{t}}=\frac{2 n-1}{2 \sqrt{t}} .
\end{aligned}
$$

Combining (6.7), (6.9)-(6.12) we can rewrite (1.19) in the form

$$
2 t \frac{d u}{d t}+\sqrt{t} u_{r}+\frac{2 n+1}{2} u=\frac{2 n-1}{2} \frac{1}{\sqrt{t}} .
$$

Since

$$
2 t \frac{d u}{d t}=2 t\left(u_{t}+u_{r} \frac{d r}{d t}\right)=2 t u_{t}+\sqrt{t} u_{r}
$$

we can rewrite $(6.13)$ also in the form

$$
t u_{t}+\sqrt{t} u_{r}+\frac{2 n+1}{4} u=\frac{2 n-1}{4} \frac{1}{\sqrt{t}} \quad \text { on }|x|=\sqrt{t} .
$$

The differential equation for $u$ is

$$
u_{t}-u_{r r}-\frac{n-1}{r} u_{r}=0 \text {. }
$$

We wish to analyze the effect of the approximation $\theta_{0}+\varepsilon u$ on the water region. To do this it will be convenient to perform a change of variables

$$
t=e^{s}, \quad r=y e^{s / 2}
$$

and work with the dependent function

$$
w(y, s)=r u(r, t) .
$$

By direct calculation we find that

$$
\begin{gathered}
w_{s}-w_{y y}-\left(\frac{n-3}{y}+\frac{y}{2}\right) w_{y}+\frac{n-3}{y^{2}} w=0 \quad \text { in } \Omega, \\
w_{s}+\frac{1}{2} w_{y}+\frac{2 n-3}{4} w=\frac{2 n-1}{4} \text { on } y=1, s>0, \\
w=0 \text { on the remaining part of } \partial \Omega,
\end{gathered}
$$


where

$$
\Omega=\left\{(y, s) ; \alpha e^{-s / 2}<y<1,0<s<\infty\right\}
$$

Set

$$
\Omega_{T}=\Omega \cap\{s<T\}, \quad \text { for any } T>0 .
$$

In the sequel we shall use parabolic Schauder estimates with norm

$$
\|w\|_{2+\beta}^{\Omega_{T}} \equiv\left\|w_{t}\right\|_{\beta}^{\Omega_{T}}+\sum_{j=0}^{2}\left\|D_{y}^{j} w\right\|_{\beta}^{\Omega_{T}}+H_{(1+\beta) / 2}^{t}\left(D_{y} w\right)
$$

where $\|v\|_{\beta}$ is the $\beta$-Hölder norm with respect to the distance function

$$
d((y, s),(\bar{y}, \bar{s}))=\left\{|y-\bar{y}|^{2}+|s-\bar{s}|\right\}^{1 / 2}
$$

and $H_{(1+\beta) / 2}^{t}(v)$ is the Hölder coefficient of $v$ in the $t$ variable (with the usual distance function) of exponent $(1+\beta) / 2$. We shall also use the "interior" norm $\widehat{\|w\|_{2+\beta}} \Omega_{T}$ whereby $\left\|D_{y}^{j} w\right\|_{\beta}^{\Omega_{T}}$ is replaced by

$$
{\widehat{\left\|D_{y}^{j} w\right\|_{\beta}}}_{\Omega_{T}}=\sup s^{j}\left|D_{y}^{j} w(y, s)\right|+\sup s^{j+\beta / 2} \frac{\left|D_{y}^{j} w(y, s)-D_{y}^{j} w(\bar{y}, \bar{s})\right|}{|y-\bar{y}|^{\beta}+|s-\bar{s}|^{\beta / 2}}
$$

$\left((y, s) \in \Omega_{T},(\bar{y}, \bar{s}) \in \Omega_{T}\right.$ and $\left.0<\bar{s}<s\right)$, and similarly for $D_{t} w$ (with the factor $s^{j}$ replaced by $\left.s^{2}\right)$ and $H_{(1+\beta) / 2}^{t}\left(D_{y} w\right)$.

We denote the space of functions with finite norm \|\|$_{2+\beta}^{\Omega_{T}}$ by $C^{2+\beta}\left(\overline{\Omega_{T}}\right)$.

Theorem 6.1. There exists a unique solution $w$ of (6.17)-(6.19) such that

$$
w \in C\left(\overline{\boldsymbol{\Omega}}_{T}\right) \cap C^{2+\beta}\left(\overline{\boldsymbol{\Omega}}_{T} \backslash \overline{\boldsymbol{\Omega}}_{\lambda}\right)
$$

for any $0<\lambda<T<\infty$.

By going back to the original variables we deduce that the unique weak solutiuon of $(2.1)-(2.3)$ is in $C^{2+\beta}$ in $\bar{G}_{T} \backslash \bar{G}_{\lambda}$ for any $0<\lambda<T<\infty$ and in $C\left(\bar{G}_{T}\right)$.

Proof. Let $\rho(s)$ be a smooth function, $0 \leq \rho \leq 1, \rho(0)=0, \rho(s)=1$ for $s \geq 1,0 \leq \rho^{\prime} \leq 2$, and set

$$
\rho_{\varepsilon}(s)=\rho\left(\frac{s}{\varepsilon}\right) \quad \text { for any } \varepsilon>0 .
$$

Consider the problem $\left(P_{\varepsilon}\right)$ consisting of $(6.17),(6.19)$ and

$$
w_{s}+\frac{1}{2} w_{y}+\frac{2 n-3}{4} w=\frac{2 n-1}{4} \rho_{\varepsilon}(s) \text { on } y=1, s>0 .
$$

For this problem the consistency condition is satisfied at $y=1, s=0$.

Let

$$
X_{\delta}=\left\{w \in C^{2+\beta}\left(\bar{\Omega}_{\delta}\right), w=0 \text { on } s=0 \text { and on } y=\alpha e^{-s / 2}\right\}
$$


For any $w \in X_{\delta}$ let $\bar{w}$ be the solution of (6.17), (6.19) with

$$
\bar{w}(1, s)=E(w)(s) \equiv \int_{0}^{s}\left[\frac{2 n-1}{4} \rho_{\varepsilon}(\tau)-\frac{1}{2} w_{y}(1, \tau)-\frac{2 n-3}{4} w(1, \tau)\right] d \tau .
$$

Since $w_{y}$ is Hölder continuous in $s$ of exponent $(1+\beta) / 2$, we easily find that

$$
\|E(w)\|_{1+\beta / 2} \leq C_{n}\left(\frac{\delta^{1-\beta / 2}}{\varepsilon}+\delta^{1 / 2}\|w\|_{2+\beta}^{\Omega_{\delta}}\right) .
$$

Hence $\bar{w} \in X_{\delta}$. Set $\bar{w}=U w$. If $w_{1}, w_{2}$ belong to $X_{\delta}$ then estimating

$$
\left\|E\left(w_{1}\right)-E\left(w_{2}\right)\right\|_{1+\beta / 2} \leq C_{n} \delta^{1 / 2}\left\|w_{1}-w_{2}\right\|_{2+\beta}^{\Omega_{\delta}}
$$

and using Schauder's estimates $[5,13]$ we deduce that

$$
\left\|U w_{1}-U w_{2}\right\|_{2+\beta}^{\Omega_{\delta}} \leq C_{n} \delta^{1 / 2}\left\|w_{1}-w_{2}\right\|_{2+\beta}^{\Omega_{\delta}}, \quad C_{n} \text { constant (depending on } n \text { ). }
$$

Therefore, if $\delta$ is sufficiently small then $U$ is a contraction, and consequently, it has a unique fixed point $w_{\varepsilon} ; w_{\varepsilon}$ is the solution of $(6.17),(6.19),(6.20)$. To extend the solution for all $t>0$, set

$$
m(t)=\left\|w_{\varepsilon}\right\|_{2+\beta}^{\Omega_{t}} .
$$

Proceeding as in the first step we can derive the estimate

$$
m(t+\delta) \leq \gamma m(t)+\gamma_{0} \delta^{1 / 2} m(t+\delta)
$$

where $\gamma, \gamma_{0}$ are positive constants independent of $t, \delta$. Choosing $\delta$ such that $\gamma_{0} \delta^{1 / 2}=\frac{1}{2}$ we deduce the estimate $m(t+\delta) \leq 2 \gamma m(t)$ which allows us to extend the solution $w$ step-by-step for all $t>0$.

If in the first step we use the interior norm \|\| mentioned above, then instead of (6.21) we get

$$
{\widehat{\|E(w)\|_{1+\beta / 2}}} \leq C+\delta^{1 / 2}{\widehat{\left\|w_{\varepsilon}\right\|_{2+\beta}}}_{\Omega_{\delta}}, \quad C \text { independent of } \varepsilon .
$$

The mapping $U$ has the unique fixed point $w_{\varepsilon}$ as before, and

$$
\widehat{\left\|w_{\varepsilon}\right\|_{2+\beta}^{\Omega_{\delta}} \leq C_{0}}
$$

where $C_{0}$ and $\delta$ are independent of $\varepsilon$.

We now let $\varepsilon \rightarrow 0$. First observe, by the maximum principle, that

$$
0 \leq w_{\varepsilon^{\prime}} \leq w_{\varepsilon} \text { if } 0<\varepsilon<\varepsilon^{\prime} .
$$

Hence $w=\lim w_{\varepsilon}$ exists. Secondly, by (6.23) and (6.22),

$$
\widehat{\|w\|_{2+\beta}} \Omega_{\delta} \leq C_{0}, \quad\|w\|_{2+\beta}^{\Omega_{T} \backslash \Omega_{\delta / 2}} \leq C_{T} \quad \forall T<\infty .
$$

Since $w$ clearly satisfies $(6.17)-(6.19)$, it remains to show that $w \in C\left(\bar{\Omega}_{T}\right)$, or just that

$$
\lim _{s \rightarrow 0} w(y, s)=0
$$


But the function $(2 n-1) s / 4$ is a supersolution of (6.17) in any $\Omega_{T}$ for $n \geq 3$ and in $\Omega_{T}$ ( $T$ small) if $n=2$. By the maximum principle we find that

$$
w(y, s) \leq \frac{2 n-1}{4} s \text { in } \Omega_{T},
$$

and thus (6.25) follows.

Definition 6.1. We depict two subsets on the free boundary $\Gamma_{\infty}: \Gamma^{+}=\{u>\kappa\}$, $\Gamma^{-}=\{u<\kappa\}$.

From (1.15), (1.16) we see that $R>0$ on $\Gamma^{+}$and $R<0$ on $\Gamma^{-}$. This means that the approximate free boundary $x=z+\varepsilon \zeta$ expands near $\Gamma^{+}$and shrinks near $\Gamma^{-}$, i.e.,

$$
\begin{aligned}
& \text { small surface tension increases the water region } \\
& \text { near } \Gamma^{+} \text {and decreases it near } \Gamma^{-} \text {. }
\end{aligned}
$$

We now state the main result of this section.

Theorem 6.2. For any $0<\eta<1$ the solution $w(y, s)$ of (6.17)-(6.19) satisfies

$$
\lim _{s \rightarrow \infty} w(y, s)=w_{\infty}(y) \quad \text { uniformly in } y, \eta \leq y \leq 1
$$

where $w_{\infty}$ is the solution of (5.1)-(5.3) asserted in Theorems 5.1 and 5.2.

Corollary 6.3. The limit $w_{\infty}(1)=\lim _{s \rightarrow \infty} w(1, s)$ exists and $w_{\infty}(1)>1$.

In view of $(6.16)$ this means that

$$
u>\kappa \text { on } \Gamma_{\infty} \mid \Gamma_{t_{0}} \text { for some large } t_{0}>0,
$$

i.e., the effect of the surface tension is to increase the water region at all times $t>t_{0}$. The amount of increase is determined by the interface $x=z+\varepsilon \zeta$. Asymptotically,

$$
\zeta=2(u-\kappa) \sqrt{t} \nu_{0} \approx 2\left(w_{\infty}(1)-1\right) \nu_{0},
$$

which means that the radial increase due to surface tension is, at time $t$,

$$
\approx 2 \varepsilon\left(w_{\infty}(1)-1\right) / \sqrt{t} \text { times the original radius. }
$$

Notice also that by $(6.25)$,

$$
u<\kappa \text { on } \Gamma_{t_{1}}
$$

for $t_{1}$ sufficiently small, i.e., the surface tension decreases the water region at all small times.

For $n \geq 3$ the operator $\mathscr{L}_{n}$ satisfies the conditions for the maximum principle. This makes the proof of Theorem 6.2 simpler. It will be convenient to first give the proof in this case, and then establish it separately for $n=2$.

Proof of Theorem 6.2 in case $n \geq 3$. Comparing $w(x, s)$ with $w_{\infty}(x)$ in $\Omega$ we immediately find that

$$
w(y, s) \leq w_{\infty}(y) \text { in } \Omega .
$$


Given any $0<\varepsilon<1$, let

$$
\widetilde{\Omega}_{\varepsilon}=\left\{(x, s) ; \varepsilon<y<1, s>s_{\varepsilon}\right\}, \quad s_{\varepsilon}=\log \frac{\alpha^{2}}{\varepsilon^{2}} ;
$$

notice that $\widetilde{\Omega}_{\varepsilon}=\Omega \cap\left\{y>\varepsilon, s>s_{\varepsilon}\right\}$ and $\left(\varepsilon, s_{\varepsilon}\right) \in \partial \Omega$. Consider the problem

$$
\begin{aligned}
& \frac{\partial w}{\partial s}+\mathscr{L}_{n} w=0 \text { in } \widetilde{\Omega}_{\varepsilon}, \\
& \left(\widetilde{P}_{\varepsilon}\right) \quad \frac{\partial w}{\partial s}+\frac{1}{2} \frac{\partial w}{\partial y}+\frac{2 n-3}{4} w=\frac{2 n-1}{4} \text { on } y=1, \\
& w=0 \text { on the remaining boundary of } \widetilde{\Omega}_{\varepsilon} .
\end{aligned}
$$

As in the proof of Theorem 6.1 , this problem has a unique solution $\tilde{w}_{\varepsilon}$, continuous on $\overline{\widetilde{\Omega}}_{\varepsilon}$ and in $C^{2+\beta}$ in $\overline{\widetilde{\Omega}}_{\varepsilon} \cap\left\{s>s_{\varepsilon}\right\}$. By the maximum principle,

$$
0 \leq \tilde{w}_{\varepsilon} \leq w \text { in } \tilde{\Omega}_{\varepsilon} .
$$

By the maximum principle we also have

$$
\tilde{w}_{\varepsilon}(y+h, s) \geq \tilde{w}_{\varepsilon}(y, s) \quad(h>0),
$$

so that

$$
\frac{\partial \tilde{w}_{\varepsilon}}{\partial s} \geq 0
$$

We claim that

$$
\frac{\partial \tilde{w}_{\varepsilon}}{\partial s} \leq A e^{-\lambda s} \quad \text { for some } A>0, \lambda>0 ;
$$

$A$ and $\lambda$ may depend on $\varepsilon$. Once this is proved, it follows that

$$
\tilde{w}_{\varepsilon}(y, s) \rightarrow w_{\varepsilon}(y) \text { as } s \rightarrow \infty,
$$

uniformly in $y$, and therefore, by (6.29),

$$
\lim _{s \rightarrow \infty} w(y, s) \geq w_{\varepsilon}(y)
$$

uniformly in $y$. Recalling that $w_{\varepsilon}(y) \rightarrow w_{\infty}(y)$, we then deduce that, for any $0<\eta<1$,

$$
\lim _{s \rightarrow \infty} w(y, s) \geq w_{\infty}(y)
$$

uniformly in $y, \eta \leq y \leq 1$. In view of (6.28), the proof of (6.27) is then complete. Thus it remains to prove $(6.31)$.

Note that

$$
\frac{\partial \tilde{w}_{\varepsilon}}{\partial s} \leq \tilde{A}_{\varepsilon} \quad \text { if } \varepsilon \leq y \leq 1, s=s_{\varepsilon}+1
$$

For $n \geq 4$ the function $W \equiv e^{-\lambda s}$ satisfies

$$
W_{s}+\mathscr{L}_{n} W \geq 0 \text { in } \widetilde{\Omega}_{\varepsilon},
$$




$$
W_{s}+\frac{1}{2} W_{y}+\frac{2 n-3}{4} W \geq 0 \text { at } y=1
$$

provided $\lambda=\min \{n-3,(2 n-3) / 4\}$. Applying the maximum principle to

$$
\zeta \equiv \frac{\partial \tilde{w}_{\varepsilon}}{\partial s}-A W, \quad A=\tilde{A}_{\varepsilon} e^{\lambda\left(s_{\varepsilon}+1\right)}
$$

in $\widetilde{\Omega}_{\varepsilon} \cap\left\{s>s_{\varepsilon}+1\right\}$ we deduce that $\zeta \leq 0$, and (6.31) follows.

Now let $n=3$. Setting

$$
\alpha_{0}=\int_{0}^{1} e^{\left(1-z^{2}\right) / 4} d z>1,
$$

we know from Theorem 5.1 that

$$
\begin{aligned}
& w_{\infty}(y)=\frac{5}{2+3 \alpha_{0}} \int_{0}^{y} e^{\left(1-z^{2}\right) / 4} d z \\
& w_{\infty}(1)=\frac{5 \alpha_{0}}{2+3 \alpha_{0}}>1 .
\end{aligned}
$$

Further, for small $\gamma>0$, if $W_{\gamma}$ is the solution of

$$
\begin{aligned}
W_{y y}+\frac{y}{2} W_{y}+\gamma W & =0, & 0<y<1, \\
\frac{1}{2} W_{y}+\frac{3-4 \gamma}{4} W & =\frac{5}{4}, & y=1, \\
W(0) & =0, &
\end{aligned}
$$

then $W_{\gamma}(y)>0$ if $\varepsilon \leq y \leq 1$ provided $\gamma$ is sufficiently small. We can now apply the maximum principle to $\zeta=\partial \tilde{w}_{\varepsilon} / \partial s-A e^{-\gamma s} W_{\gamma}(y)$ and deduce that $\zeta \leq 0$ in $\widetilde{\Omega}_{\varepsilon} \cap\left\{s>s_{\varepsilon}+1\right\}$, and the assertion (6.31) follows.

Proof of Theorem 5.2 in case $n=2$. The functions

$$
e^{-\gamma s} w(y, s) \text { and } e^{-\gamma s} w_{\infty}(y)
$$

satisfy the same parabolic equation in $\Omega_{T}$ :

$$
W_{s}+\mathscr{L}_{2} W+\gamma W=0
$$

with positive lowest order coefficient if

$$
\gamma=\frac{1}{y_{0}^{2}} \quad \text { where } y_{0}=\alpha e^{-T / 2}
$$

Applying the maximum principle we conclude that $e^{-\gamma s} w \leq e^{-\gamma s} w_{\infty}$ in $\Omega_{T}$, i.e., $w \leq w_{\infty}$ in $\Omega_{T}$. Since $T$ is arbitrary,

$$
w \leq w_{\infty} \text { in } \Omega \text {. }
$$

Next we define $\tilde{w}_{\varepsilon}$ as in case $n \geq 3$. Applying the maximum principle to

$$
\zeta=e^{-\gamma s} \tilde{w}_{\varepsilon}
$$

and to

$$
\zeta=e^{-\gamma s}\left(\tilde{w}_{\varepsilon}-w\right)
$$


in $\widetilde{\Omega}_{\varepsilon}$, where $\gamma=1 / \varepsilon^{2}$, we deduce that

$$
0 \leq \tilde{w}_{\varepsilon} \leq w \text { in } \widetilde{\Omega}_{\varepsilon} .
$$

Similarly we deduce that

$$
\zeta=\tilde{w}_{\varepsilon}(y, s+h) e^{-\gamma(s+h)}-\tilde{w}_{\varepsilon}(y, s) e^{-\gamma s}
$$

is $\geq 0$ in $\widetilde{\Omega}_{\varepsilon}$ and, consequently,

$$
\frac{\partial \tilde{w}_{\varepsilon}}{\partial s} \geq 0 \text { in } \tilde{\Omega}_{\varepsilon} .
$$

Recalling (6.32), it follows that $\partial \tilde{w}_{\varepsilon} / \partial s \rightarrow 0$ "weakly" as $s \rightarrow \infty$, i.e.,

$$
\int_{\Sigma}^{\Sigma+1} \frac{\partial \tilde{w}_{\varepsilon}(y, s)}{\partial s} d s \rightarrow 0 \text { if } \Sigma \rightarrow \infty, \text { for all } \varepsilon \leq y \leq 1
$$

Set

$$
\widetilde{W}_{\varepsilon}(y, s)=\int_{s}^{s+1} \tilde{w}_{\varepsilon}\left(y, s^{\prime}\right) d s^{\prime}
$$

Then

$$
\begin{gathered}
\frac{\partial \widetilde{W}_{\varepsilon}}{\partial s}+\mathscr{L}_{2} \widetilde{W}_{\varepsilon}=0 \quad \text { in } \widetilde{\Omega}_{\varepsilon}, \\
\frac{\partial \widetilde{W}_{\varepsilon}}{\partial s}+\frac{1}{2} \frac{\partial \widetilde{W}_{\varepsilon}}{\partial y}+\frac{1}{4} \widetilde{W}_{\varepsilon}=\frac{3}{4}, \quad y=1, \quad s>s_{\varepsilon}, \\
\widetilde{W}_{\varepsilon}(\varepsilon, s)=0, \quad s>s_{\varepsilon}, \\
\widetilde{W}_{\varepsilon}\left(y, s_{\varepsilon}\right) \geq 0, \quad \varepsilon \leq y \leq 1 .
\end{gathered}
$$

Further, by (6.32) and $w_{\infty}(y) \leq w_{\infty}(1) \leq \frac{3}{2}$, and (6.35),

$$
\begin{gathered}
0 \leq \widetilde{W}_{\varepsilon} \leq \frac{3}{2}, \quad 0 \leq \frac{\partial \widetilde{W}_{\varepsilon}}{\partial s} \leq \frac{3}{2} \\
\frac{\partial \widetilde{W}_{\varepsilon}}{\partial s} \rightarrow 0 \quad \text { as } s \rightarrow \infty, \text { for each } y \in(\varepsilon, 1) .
\end{gathered}
$$

It follows that $\widetilde{W}_{\varepsilon}(y, s) \uparrow w_{\varepsilon}(y)$ as $s \uparrow \infty$ where $w_{\varepsilon}$ is a solution to problems $\left(P_{\varepsilon}^{\infty}\right)(n=2)$, and $w_{\varepsilon}(y)>0$ if $\varepsilon<y<1$. The convergence is uniform in $y$, by Dini's theorem.

Noting that $\widetilde{W}_{\varepsilon}(y, s) \leq \tilde{w}_{\varepsilon}(y, s+1)$ and recalling $(6.33)$, we get

$$
\varliminf_{s \rightarrow \infty} w(y, s) \geq w_{\varepsilon}(y), \quad \varepsilon \leq y \leq 1 \text {. }
$$

Finally, letting $\varepsilon \rightarrow 0$ and recalling also (6.32), the assertion (6.27) follows. 


\section{THE EFFECT OF SURFACE TENSION ON THE INTERFACE (GENERAL CASE)}

In this section we shall extend the results of $\S 6$ to general shapes of $D$ and $G$, showing that (small) surface tension increases the water region for large $t$; the fact that small surface tension decreases the water region for small $t$ is valid whenever $u$ can be shown to be continuous in $\overline{G_{t}}$ for some $t_{*}>0$.

If

$$
0<c_{1} \leq \int_{\partial D} \tilde{\theta}(x, t) \leq c_{2}<\infty \text { for all } t \geq 0
$$

then (Matano [15]) there exists a positive number $T_{0}$ such that the free boundary has the form (1.10) with $\rho \in C^{\infty}$ for all $t \geq T_{0}$. We shall also assume in this section that (1.11) holds for some $T>T_{0}$. Finally we assume that, for some $T^{*}>T$,

$$
\begin{aligned}
& \left.\tilde{\theta}\right|_{\partial D_{\nsim}} \text { is independent of } t, \text { if } t>T^{*}, \\
& \text { and, if } n=2,\left.\tilde{\theta}\right|_{\partial D_{\nsim}} \text { is constant if } t>T^{*} .
\end{aligned}
$$

Under this additional assumption it was proved by Matano [15] that the function $\rho(\theta, t)$ satisfies

$$
\left|\rho(\theta, t)-M t^{1 / 2}\right| \leq C_{0} \quad \text { if } t \geq t_{0}
$$

where $M, C_{0}, t_{0}$ are positive constants. From regularity results for the free boundary (cf. [6]) we further deduce that, for any $j>0$,

$$
\left|D^{j}\left(\rho(\theta, t)-M t^{1 / 2}\right)\right| \leq C_{j} \quad \text { if } t>t_{0} .
$$

We can therefore perform a change of variable $x \rightarrow \xi$ such that, for $t>t_{0}$, the free boundary becomes $|\xi|=M t^{1 / 2}$ whereas the derivatives $\partial x_{i} / \partial \xi_{j}$ are $\delta_{i j}+$ $O\left(t^{-1 / 2}\right)$. Let us perform another change of variables $\xi \rightarrow\left(y, \varphi_{1}, \ldots, \varphi_{n-1}\right)$, $t \rightarrow s$, where

$$
t=e^{s}, \quad y=|\xi| e^{s / 2}
$$

and $\varphi_{1}, \ldots, \varphi_{n-1}$ are the spherical coordinates of $\xi$. Then the function

$$
u\left(y, \varphi_{1}, \ldots, \varphi_{n-1}, s\right)=|\xi| u
$$

satisfies (we take for simplicity $M=1$ ),

$$
w_{s}+\widetilde{L}_{n} w \equiv w_{s}-w_{y y}+\mathscr{M} w-\left(\frac{n-3}{y}+\frac{y}{2}\right) w_{y}+\frac{n-3}{y^{2}} w=0 \quad \text { in } \Omega,
$$

$$
w_{s}+\frac{1}{2} w w_{y}+\mathcal{N} w+\frac{2 n-3}{4} w=\frac{2 n-1}{4} \text { at } y=1
$$

where

$$
\mathscr{M} w=\sum a_{i} \frac{\partial^{2} w}{\partial \eta_{1} \partial \eta_{1}}+\sum h_{1} \frac{\partial w}{\partial \eta_{i}},
$$




$$
\mathscr{N} w=\sum c_{i} \frac{\partial w}{\partial \eta_{i}}, \quad\left(\eta_{1}, \ldots, \eta_{n}\right)=\left(y, \varphi_{1}, \ldots, \varphi_{n-1}\right)
$$

and

$$
\sum\left|a_{i j}\right|+\sum\left|b_{i}\right|+\sum\left|c_{i}\right| \leq \sigma(s), \quad \sigma(s) \rightarrow 0 \quad \text { if } s \rightarrow \infty .
$$

Equations (7.5), (7.6) are taken of course in the weak sense corresponding to the definition of the weak solution $u$.

Theorem 7.1. For any $0<\eta<1$ the solution $w$ satisfies a.e.

$$
w(y, s) \rightarrow w_{\infty}(y) \text { as } s \rightarrow \infty \text {, uniformly in } y, \eta \leq y \leq 1,
$$

where $w_{\infty}(y)$ is the solution of (5.1)-(5.3), as described in Theorems 5.1 and 5.2 .

Proof. Set

$$
\mathscr{L}_{n} w \equiv-w_{y y}-\left(\frac{n-3}{y}+\frac{y}{2}\right) w_{y}+\frac{n-3}{y^{2}} w
$$

and consider first the case $n \geq 3$. Let $\tilde{w}_{\varepsilon, \delta} \quad(\delta>0)$ denote the solution of

$$
\begin{gathered}
\frac{\partial w}{\partial s}+\mathscr{L}_{n} w=-\delta \quad \text { in } \widehat{\Omega}_{\varepsilon} \equiv\left\{\varepsilon<y<1, s>\sigma_{\varepsilon}\right\}, \\
\frac{\partial w}{\partial s}+\frac{1}{2} \frac{\partial w}{\partial y}+\frac{2 n-3}{4} w=\frac{2 n-1}{4}-\delta \quad \text { on } y=1, \\
w=0 \text { on }\left\{y=\varepsilon, s>\sigma_{\varepsilon}\right\} \quad \text { and on } \quad\left\{\varepsilon<y<1, s=\sigma_{\varepsilon}\right\}
\end{gathered}
$$

where $\sigma_{\varepsilon}$ is sufficiently large, depending on $\delta$. As in the proof of (6.31) one can show that

$$
\zeta= \pm \frac{\partial \tilde{w}_{\varepsilon, \delta}}{\partial s}-A W \leq 0 \text { in } \widehat{\Omega}_{\varepsilon}
$$

and thus

$$
\left|\frac{\partial \tilde{w}_{\varepsilon, \delta}}{\partial s}\right| \leq A e^{-\lambda s} \quad(\lambda>0)
$$

It follows that

$$
\tilde{w}_{\varepsilon, \delta}(y, s) \rightarrow w_{\varepsilon, \delta}(y) \quad \text { as } s \rightarrow \infty
$$

From (7.7) we deduce that if $\sigma_{\varepsilon}$ is sufficiently large then $\tilde{w}_{\varepsilon, \delta}$ satisfies

$$
\begin{gathered}
\frac{\partial w}{\partial s}+\widetilde{\mathscr{L}}_{n} w \leq 0 \quad \text { in } \widehat{\Omega}_{\varepsilon}, \\
\frac{\partial w}{\partial s}+\frac{1}{2} \frac{\partial w}{\partial y}+\mathscr{N} w+\frac{2 n-3}{4} w \leq \frac{2 n-1}{4} \quad \text { on } y=1 .
\end{gathered}
$$

If $w(y, s)$ were smooth then by the maximum principle we could deduce that

$$
\tilde{w}_{\varepsilon, \delta}(y, s) \leq w(y, s)+A W
$$

where $W$ is the function used in the proof of (6.31), with smaller $\lambda$ if $n \geq 4$ 
and slightly modified when $n=3$ :

$$
W=e^{-\gamma s} \widehat{W}_{\gamma}(y)
$$

where

$$
\begin{gathered}
\mathscr{L}_{3} \widehat{W}_{\gamma}-\gamma \widehat{W}_{\gamma}=\gamma, \quad 0<y<1, \\
\widehat{W}_{\gamma}(0)=0, \quad \frac{1}{2} \widehat{W}_{\gamma}^{\prime}(1)+\frac{3-4 \gamma}{4} \widehat{W}_{\gamma}(1)=\gamma ;
\end{gathered}
$$

with this modification,

$$
\begin{gathered}
W_{s}+\widetilde{\mathscr{L}}_{n} W \geq 0 \text { in } \widehat{\Omega}_{\varepsilon}, \\
\frac{\partial W}{\partial s}+\frac{1}{2} \frac{\partial W}{\partial y}+\mathcal{N} W+\frac{2 n-3}{4} W \geq 0 \quad \text { on } y=1
\end{gathered}
$$

not only for $n \geq 4$ but also for $n=3$.

From (7.13) we deduce that

$$
\varliminf_{s \rightarrow \infty} w(y, s) \geq w_{\varepsilon, \delta}(y) .
$$

Since further

$$
w_{\varepsilon, \delta}(y) \rightarrow w_{\varepsilon}(y) \quad \text { if } \delta \rightarrow 0
$$

and

$$
w_{\varepsilon}(y) \rightarrow w_{\infty}(y) \quad \text { if } \varepsilon \rightarrow 0
$$

it follows that

$$
\varliminf_{s \rightarrow \infty} w(y, s) \geq w_{\infty}(y)
$$

provided $w$ is smooth.

For the actual weak solution $w$ the inequality (7.13) can be established by working with finite differences, and then (7.15) follows provided " $\underline{\text { lim }}$ " is taken in the "essential lim inf" sense.

It remains to prove that

$$
\varlimsup_{s \rightarrow \infty} w(y, s) \leq w_{\infty}(y) .
$$

(The uniform convergence in $y$ asserted in Theorem 7.1 follows from the estimates from which (7.15), (7.16) are derived.)

Let $\hat{w}_{\varepsilon, \delta}$ be the solution of

$$
\begin{gathered}
\mathscr{L}_{n} w=\delta, \quad \varepsilon<y<1, \\
\frac{1}{2} w^{\prime}(1)+\frac{2 n-3}{4} w(1)=\frac{2 n-1}{4}+\delta, \\
w(\varepsilon)=0,
\end{gathered}
$$

for any $0<\delta<1$. Clearly

$$
\hat{w}_{\varepsilon, \delta} \geq 0
$$


and we also have an upper bound

$$
\hat{w}_{\varepsilon, \delta} \leq C
$$

where $C$ is a constant independent of $\varepsilon, \delta$. Indeed, if $n \geq 4$ then $C=$ $1+(2 n-1) /(2 n-3)$ is a supersolution of $(7.17)-(7.19)$ if $\delta<1$, whereas if $n=3$ we can take $C-y^{2}$ as a supersolution provided $C$ is a sufficiently large positive constant.

From $(7.20),(7.21)$ and a compactness argument, $\lim _{\varepsilon \rightarrow 0} \hat{w}_{\varepsilon, \delta}(y)=\hat{w}_{\delta}(y)$ exists $(0<y \leq 1)$ for a sequence $\varepsilon \rightarrow 0$, and $\hat{w}_{\delta}$ satisfies $(7.17)$ with $\varepsilon=0$, and (7.18). Since $\zeta \equiv C y^{\lambda}$ is a supersolution (i.e. $\mathscr{L}_{n} \zeta \geq \delta$ ) in $0<y<y_{0}$ for some constants $C>0,0<\lambda<1$ and small enough $y_{0}$, we have (cf. (5.10)) $\hat{w}_{\delta}(y) \leq C y^{\lambda}$. It follows that $\lim _{\delta \rightarrow 0} \hat{w}_{\delta}(y) \equiv \hat{w}(y) \quad(0<y \leq 1)$ exists for some sequence $\delta \rightarrow 0$, and $\hat{w}$ satisfies (5.1)-(5.3). By uniqueness, $\hat{w} \equiv w_{\infty}$, i.e.,

$$
\hat{w}_{\delta}(y) \downarrow w_{\infty}(y) \text { if } \delta \downarrow 0 \quad(0<y \leq 1) .
$$

Arguing as in the proof of (7.13) we find by comparison that

$$
w(y, s) \leq \hat{w}_{\delta}(y)+A W \quad \text { in } \Omega \cap\left\{s>\hat{\sigma}_{\varepsilon}\right\} .
$$

Letting $s \rightarrow \infty$ we get

$$
\varlimsup_{s \rightarrow \infty} w(y, s) \leq \hat{w}_{\delta}(y)
$$

and together with (7.22), the assertion (7.16) follows in case $n \geq 3$.

The proof for $n=2$ requires some modifications. For any $\varepsilon \in(0,1)$, if $\lambda$ is a sufficiently small positive number then, by standard ODE arguments there exists a positive solution $w=w_{\varepsilon, \lambda}(y)$ of

$$
\begin{gathered}
\mathscr{L}_{2} w-\lambda w=0, \quad \varepsilon<y<1, \\
\frac{1}{2} w^{\prime}(1)+\frac{1}{4} w(1)-\lambda w(1)=\frac{3}{4}, \\
w(\varepsilon)=0 .
\end{gathered}
$$

We can then apply the maximum principle to

$$
\zeta \equiv\left( \pm \frac{\partial \tilde{w}_{\varepsilon, \delta}}{\partial s}-A e^{-\lambda s} w_{\varepsilon, \lambda}\right) e^{\gamma s}, \quad \gamma=\frac{1}{\varepsilon^{2}}
$$

in $\widehat{\Omega}_{\varepsilon}$ to deduce that $\zeta \leq 0$; hence,

$$
\left|\frac{\partial \tilde{w}_{\varepsilon, \delta}}{\partial s}\right| \leq A e^{-\lambda s} \quad(\lambda=\lambda(\varepsilon)) .
$$

It follows that (7.12) holds and then also (7.14) is valid, for any small $\delta>0$. The assertion (7.15) now follows from (7.14), as before.

To prove (7.16) we can establish for $n=2$ the existence of unique positive solution $\hat{w}_{\varepsilon, \delta}$ of $(7.17)-(7.19)$, as in the case $\delta=0$, and also show that 


$$
\begin{aligned}
& \hat{w}_{\varepsilon, \delta} \uparrow \hat{w}_{\delta} \quad \text { as } \varepsilon \downarrow 0, \\
& \hat{w}_{\delta} \downarrow w_{\infty} \text { as } \delta \downarrow 0 .
\end{aligned}
$$

Thus it remains only to establish an estimate of the form (cf. (7.23))

$$
w(y, s) \leq \hat{w}_{\delta}(y)+A e^{-\lambda s} w(y) .
$$

This follows by comparison provided we can take $w$ a solution of

$$
\begin{gathered}
\mathscr{L}_{2} w-\lambda w=0, \quad 0<y<1, \\
\frac{1}{2} w^{\prime}(1)+\frac{1}{4} w(1)-\lambda w(1) \geq 0 .
\end{gathered}
$$

But $w$ can be constructed as a limit of solutions $w_{\varepsilon, \lambda}(\varepsilon \downarrow 0)$ by the method of proof of $\S 5$ for $n=2$ (with $\lambda=0$ ) provided $\lambda$ is small.

\section{The two-Phase Stefan PRoblem}

In this section we conisder the two-phase Stefan problem with surface tension:

$$
\begin{gathered}
\partial \theta_{1} / \partial t-\Delta \theta_{1}=0 \text { in } G_{T}^{1}, \\
\partial \theta_{2} / \partial t-\Delta \theta_{2}=0 \text { in } G_{T}^{2}, \\
\theta_{1}=\tilde{\theta}_{1} \quad \text { on } \partial D_{T}^{1} \cup\left(G_{1} \times\{0\}\right), \\
\theta_{2}=\tilde{\theta}_{2} \quad \text { on } \partial D_{T}^{2} \cup\left(G_{2} \times\{0\}\right), \\
\theta_{1}=\theta_{2}=\varepsilon \kappa \quad \text { on the free boundary } \Gamma_{T}, \\
\left(X_{t}+\nabla\left(\theta_{1}-\theta_{2}\right)\right) \cdot N=0 \text { on } \Gamma_{T}
\end{gathered}
$$

where $\tilde{\theta}_{1}>0, \tilde{\theta}_{2}<0$. Here $D_{1}, D_{2}, G_{0}, G_{1}, G_{2}$ are smooth domains satisfying:

$$
\bar{D}_{1} \subset G_{0}, \quad \bar{G}_{0} \subset D_{2}, \quad G_{1}=G_{0} \backslash \bar{D}_{1}, \quad G_{2}=D_{2} \backslash \bar{G}_{0} .
$$

It is well known that if $\varepsilon=0$ then the problem has a unique weak solution (cf. [7]) whereas if the initial values are sufficiently smooth and satisfy some consistency conditions at $\partial G_{0}$ then there exists a classical solution for some small time interval $0 \leq t \leq T[7,16]$. We shall now assume that a classical solution for $\varepsilon=0$ exists in some time interval $0 \leq t \leq T$, and denote it by $\left(\theta_{01}, \theta_{02}, \Gamma_{T}\right)$. We consider for small $\varepsilon>0$ the approximate solution $\left(\theta_{01}+\varepsilon u_{1}, \theta_{02}+\varepsilon u_{2}\right)$ with $u_{1}$ defined in the water region $G_{T}^{1}$ and $u_{2}$ defined in the ice region $G_{T}^{2}$. We shall derive a parabolic system for $u_{1}, u_{2}$ and then prove that it has a unique weak solution.

Introducing the notation (1.9) for $\Gamma_{T}$ and denoting by $N=N_{0}+\varepsilon N_{1}$ the normal for the interface $x=z+\varepsilon \zeta$ of the $\varepsilon$-perturbed new free boundary, we find as in $\S 1$, that if $R$ is defined by (1.15) then

$$
R(s, t)=\frac{\kappa-u_{i}}{\nabla \theta_{0 i} \cdot N_{0}}=h_{i}\left(u_{i}-\kappa\right), \quad h_{i}=-\frac{1}{\nabla \theta_{0 i} \cdot N_{0}}>0 .
$$


Further, analogously to (1.17) we get

$$
\begin{aligned}
& N_{0} \cdot N_{0} R_{t}+\sum\left(z_{t}+\nabla\left(\theta_{01}-\theta_{02}\right)\right) \cdot A_{j} N_{0} R_{s_{j}} \\
&+\left\{\left(z_{t}+\nabla\left(\theta_{01}-\theta_{02}\right)\right) \cdot \sum A_{j} N_{0, s_{j}}+N_{0} \cdot N_{0 t}+N_{0}\right. \\
&\left.\quad \cdot\left(\nabla \partial_{x_{1}}\left(\theta_{01}-\theta_{02}\right) \cdot N_{0}, \ldots, \nabla \partial_{x_{n}}\left(\theta_{01}-\theta_{02}\right) \cdot N_{0}\right)\right\} R \\
&+ \nabla\left(u_{1}-u_{2}\right) \cdot N_{0}=0 .
\end{aligned}
$$

Setting

$$
g_{i}=\nabla \theta_{0 i} \cdot N_{0}
$$

it follows from (8.2) that

$$
\left(-g_{2}\right) u_{1}=\left(-g_{1}\right) u_{2}-\kappa g \quad\left(g=g_{2}-g_{1}, g_{1}<0, g_{2}<0\right)
$$

and (cf. (1.22))

$$
a \frac{d u_{1}}{d t}+\sum b_{j} \frac{d u_{1}}{d s_{j}}+N_{0} \cdot \nabla\left(u_{1}-u_{2}\right)+c u_{1}=f_{0}
$$

where

$$
a=N_{0} \cdot N_{0} h_{1}>0
$$

and $b_{i}, c, f_{0}$ are known functions having a structure similar to (1.23).

Set

$$
G_{T}=G_{T}^{1} \cup \Gamma_{T} \cup G_{T}^{2}
$$

and extend $g_{1}, g_{2}$ and $\kappa$ smoothly into all of $\bar{G}_{T}$ in such a way that $g_{1}$ and $g_{2}$ remain negative.

Notice that the function

$$
U= \begin{cases}\left(-g_{2}\right) u_{1} & \text { in } G_{T}^{1} \\ \left(-g_{1}\right) u_{2}-\kappa g & \text { in } G_{T}^{2}\end{cases}
$$

is continuous in $G_{T}$.

To define a weak solution, we set

$$
u= \begin{cases}u_{1} & \text { in } G_{T}^{1} \\ u_{2} & \text { in } G_{T}^{2}\end{cases}
$$

and integrate by parts in

$$
\int_{i_{i}^{\prime} u i_{i}^{2}}\left(u_{t}-\Delta u\right) \varphi=0, \quad \varphi \text { smooth } .
$$

Using (8.4), (8.5) and the integration by parts formulas

$$
\begin{aligned}
\int_{\left(i_{l}^{\prime}\right)} \varphi_{t} \psi= & -\int_{\left(i_{l}^{\prime}\right)} \varphi \psi_{t}-\int_{0}^{l}(\varphi \psi)(z . l) z_{t} \cdot N_{0} d s d t \\
& +\int_{\left(i^{\prime}(l)\right.} \varphi \psi d x-\int_{\left(i^{\prime}(0)\right.} \varphi \psi d x
\end{aligned}
$$




$$
\begin{aligned}
\int_{G_{T}^{2}} \varphi_{t} \psi= & -\int_{G_{T}^{2}} \varphi \psi_{t}+\int_{0}^{T}(\varphi \psi)(z, t) z_{t} \cdot N_{0} d s d t \\
& +\int_{G^{2}(T)} \varphi \psi d x-\int_{G^{2}(0)} \varphi \psi d x
\end{aligned}
$$

we find that (cf. (2.6))

$$
\begin{gathered}
-\int_{G_{T}} u \varphi_{t}+\int_{G_{T}} \nabla \varphi \cdot \nabla u \\
-\int_{0}^{T} \int_{L} u_{1}\left\{\frac{d}{d t}(a \varphi)+\sum \frac{d}{d s_{j}}\left(b_{j} \varphi\right)-c_{1} \varphi\right\} d s d t \\
=\int_{0}^{T} \int_{L} \varphi f_{1} d s d t
\end{gathered}
$$

where

$$
c_{1}=c+\frac{g}{g_{1}} z_{t} \cdot N_{0}, \quad f_{1}=f_{0}+\frac{\kappa g}{g_{1}} z_{t} \cdot N_{0}
$$

and $\varphi$ in any function in $C^{1}\left(\bar{G}_{T}\right)$ vanishing on $G(T) \cup \partial D_{T}^{1} \cup \partial D_{T}^{2}$.

If $u_{i}$ is smooth then

$$
\begin{gathered}
\partial u_{i} / \partial t-\Delta u_{i}=0 \text { in } G_{T}^{i}, \\
u_{i}=0 \text { on } \partial D_{T}^{i} \text { and } \text { on } G_{i} \times\{0\}
\end{gathered}
$$

and (8.4), (8.5) hold on $\Gamma_{T}$. We now define a weak solution.

Definition 8.1. A function $u=\left(u_{1}, u_{2}\right)$ is a weak solution of (8.8), (8.9), (8.4), (8.5) if

$$
u_{i}, \nabla u_{i} \in L^{2}\left(G_{T}^{i}\right) \quad(i=1,2)
$$

and if (8.9) holds in the usual continuous sense, (8.4) holds in the trace sense, and (8.7) holds for any test function $\varphi$ as above.

If we substitute $\varphi=U$ in (8.7) and proceed formally, we obtain, after some integrations by parts (cf. the derivation of (3.2)) an energy estimate

$$
\begin{aligned}
& \sup _{0<t<T} \int_{G(t)} u^{2}(x, t) d x+\int_{G_{T}}|\nabla u|^{2} \\
& \quad+\sup _{0<t<T} \int_{L} u_{1}^{2}(z(s, t), t) d s \leq C_{T} .
\end{aligned}
$$

To actually use an estimate of this type in order to establish existence of a weak solution, we resort to finite-difference approximations as in $\S 3$. Instead of the elliptic problem (3.3) we now have:

$$
\frac{1}{h} u_{j}^{k}-\Delta u_{j}^{k}=\frac{1}{h} u_{j}^{k-1} \quad \text { in } G^{j, k} \quad(j=1,2),
$$


where $G^{j, k}=G^{j}(k h)$, and

$$
\begin{gathered}
\frac{1}{h} a^{k} u_{1}^{k}+N_{0}^{k} \cdot \nabla\left(u_{1}^{k}-u_{2}^{k}\right)+\sum b_{j}^{k} \frac{d u_{1}^{k}}{d s_{j}}+c_{1}^{k} u_{1}^{k} \\
=\frac{1}{h} a^{k} u^{k-1}+f_{1}^{k} \text { on } \Gamma^{k},
\end{gathered}
$$

$$
\left(-g_{2}^{k}\right) u_{1}^{k}=\left(-g_{1}^{k}\right) u_{2}^{k}-(\kappa g)^{k} \text { on } \Gamma^{k}
$$

and

$$
u_{j}^{k}=0 \quad \text { on } \partial D^{j, k}
$$

where $\partial D^{j, k}=\partial D^{j}(k h)$.

Setting

$$
U^{k}= \begin{cases}\left(-g_{2}\right)^{k} u_{1}^{k} & \text { in } G^{1, k}, \\ \left(-g_{1}\right)^{k} u_{2}^{k}-(\kappa g)^{k} & \text { in } G^{2, k},\end{cases}
$$

the system (8.11)-(8.13) can be written in the form

$$
\begin{gathered}
-\operatorname{div}\left(A^{k} \nabla U^{k}\right)+B^{k} \cdot \nabla U^{k}+\frac{1}{h} U^{k}+C^{k} U^{k}+F^{k} \\
=\sum\left(\beta_{j}^{k} \frac{\partial U^{k}}{\partial s_{j}}+\gamma U^{k}+\gamma_{0}\right) \delta
\end{gathered}
$$

where

$$
A^{k}= \begin{cases}\left(-g_{2}^{k}\right)^{-1} & \text { in } G^{1, k}, \\ \left(-g_{1}^{k}\right)^{-1} & \text { in } G^{2, k},\end{cases}
$$

and $\delta$ is the uniform mass of density 1 distributed over $\Gamma^{k}$.

Formally, if we multiply (8.15) by $U^{k}$ and integrate over $G^{k}=G^{1, k} \cup \Gamma^{k} \cup$ $G^{2, k}$, we get

$$
\begin{aligned}
\int_{G^{k}} A^{k} & \left|\nabla U^{k}\right|^{2}+\frac{1}{h} \int_{G^{k}}\left(U^{k}\right)^{2}+\int_{L}\left(U^{k}(z(s, k h), k h)\right)^{2} d s \\
\leq & \varepsilon \int_{G^{k}}\left|\nabla U^{k}\right|^{2}+C \int_{G^{k}}\left(U^{k}\right)^{2}+C \\
& +\int_{\Gamma^{k}} \gamma\left(U^{k}\right)^{2}+\int_{\Gamma^{k}} \sum \beta_{j}^{k} \frac{\partial\left(U^{k}\right)^{2}}{\partial s_{j}} \quad(\varepsilon \text { small })
\end{aligned}
$$

and the last integral is equal to

$$
-\int_{\Gamma^{k}} \sum \frac{\partial \beta_{j}^{k}}{\partial s_{i}}\left(U^{k}\right)^{2} .
$$

But for any small $\mu>0$,

$$
\int_{\Gamma^{k}}\left(U^{k}\right)^{2} \leq \frac{C}{\mu} \int_{G^{k}}\left(U^{k}\right)^{2}+\mu \int_{G^{k}}\left|\nabla U^{k}\right|^{2} .
$$


If we substitute this into (8.16) and choose $\mu$ small enough and then $h<\mu / C$, we get

$$
\int_{G^{k}}\left|\nabla U^{k}\right|^{2}+\frac{1}{h} \int_{G^{k}}\left(U^{k}\right)^{2}+\int_{L}\left(U^{k}(z(s, k h), k h)\right)^{2} d s \leq C_{T} .
$$

Notice that if we approximate $\delta$ by smooth functions $\delta_{1}^{j}(s) \delta_{2}^{j}(\lambda)$ where $\lambda$ is the distance of a point from $\Gamma^{k}$ and $\delta_{2}^{j}(\lambda) \rightarrow \delta(0)$ (=Dirac measure at 0 ), then (8.15), (8.14) becomes a diffraction problem and by known results (e.g. [13]) it has a unique solution $U^{k, j}$. Since the derivation of (8.17) is valid also for $U^{k, j}$ with a constant $C_{T}$ independent of $j$, letting $j \rightarrow \infty$ we obtain a solution $U^{k}$ of $(8.11)-(8.14)$.

We can next derive for the $U^{k}$ an a priori estimate analogous to (3.12) and use it to conclude that a subsequence of

$$
u^{h}(x, t)=u_{j}^{k}(x, k h) \quad \text { if } x \in G^{j, k}, k h \leq t<(k+1) j
$$

converges weakly to a weak solution of (8.8), (8.9), (8.4), (8.5).

Theorem 8.1. There exists a unique weak solution of (8.8), (8.9), (8.4), (8.5).

Proof. We have already proved existence. To prove uniqueness we take in the definition of weak solution the test function $\eta$ defined by

$$
-\eta_{t}=U, \quad \eta(x, T)=0 \quad(U \text { as in (8.6)); }
$$

this can be justified by approximation, as in $\S 4$. Then

$$
u_{1}=-\frac{\eta_{t}}{\left(-g_{2}\right)}, \quad u_{2}=-\frac{\eta_{t}}{\left(-g_{1}\right)}+\frac{\kappa g}{\left(-g_{1}\right)},
$$

and we can proceed as in $\S 4$, making use, in particular, of (4.9). After some integrations by parts, we end up with an inequality similar to (4.11), with the second integral on the left-hand side replaced by

$$
\int_{0}^{T} \int_{L}\left\{\frac{1}{2}|\nabla \eta|^{2} g\left[\frac{1}{\left(-g_{2}\right)}-\frac{1}{\left(-g_{1}\right)}\right]+\frac{\left|N_{0}\right|^{2}}{g_{1} g_{2}} \eta_{t}^{2}+\nabla \eta \cdot N_{0} \frac{g}{g_{1} g_{2}} \eta_{t}\right\}
$$

where we have made use of the relation $z_{t} \cdot N_{0}=g$. Since $g=g_{2}-g_{1}$, the integrand is bounded from below by $c \eta_{t}^{2}(c>0)$, and the proof can now be completed by using Gronwall's inequality.

Remark 8.1. In constructing a weak solution we used finite-difference approximation for both the parabolic equation and the boundary conditions. Actually one can use another scheme whereby the finite differencing is performed only with respect to the boundary condition on $\Gamma_{T}$. Then, for the one-phase problem we define the approximating solution by

$$
u^{h}(x, t)=u_{k}^{h}(x, t), \quad(k-1) h \leq t<k h,
$$

and replace $a \frac{d u}{d t}$ by

$$
\frac{1}{h}\left(a u_{k}^{h}\right)(z(s, t), t)-\frac{1}{h} a(z(s, t), t) u_{k}^{h}(z(s, t-h), t-h),
$$


and thus solve a parabolic equation in each interval $(k-1) h \leq t \leq k h$ with $u^{h}$ continuous across $t=k h$, for each $k$. The same procedure can be used for the two-phase problem, finite differencing only (8.5), and applying [13, Chapter IV, $\S \S 1-8]$ to prove that the corresponding parabolic system has a unique solution.

\section{THE EFFECT OF SURFACE TENSION ON THE INTERFACE}

We shall consider in this section the effect of small surface tension on the free boundary. As we shall see, in contrast to the facts established in $\S \S 6,7$ for the one-phase Stefan problem, small surface tension, for the two-phase Stefan problem, decreases the water region for all time provided the data are radial or "close" to radial.

Let $K_{i}$ be a solution of

$$
\begin{gathered}
\partial_{t} K_{i}-\Delta K_{i} \geq 0 \quad \text { on } G_{T}^{i}, \\
K_{i}=l_{i} \quad \text { on } \partial D_{T}^{i} \cup\left(G_{i} \times\{0\}\right), \\
K_{i}=\kappa \text { on } \Gamma_{T},
\end{gathered}
$$

and set $U_{i}=u_{i}-K_{i}$. Then

$$
\begin{gathered}
\partial_{t} U_{i}-\Delta U_{i} \leq 0 \quad \text { in } G_{T}^{i}, \\
U_{i}=-l_{i} \quad \text { on } \partial D_{T}^{i} \cup\left(G_{i} \times\{0\}\right), \\
g_{2} U_{1}=g_{1} U_{2} \quad \text { on } \Gamma_{T}, \\
a \frac{d U_{1}}{d t}+\sum b_{j} \frac{d U_{1}}{d s_{j}}+c U_{1}+\left(\nabla U_{1}-\nabla U_{2}\right) \cdot N_{0} \\
=\left(\nabla K_{2}-\nabla K_{1}\right) \cdot N_{0} \quad \text { on } \Gamma_{T}
\end{gathered}
$$

where $a, b_{j}, c$ are easily determined by comparing with (8.3); in particular, $a>0$.

Lemma 9.1. If $\kappa>0$ and, for some $l_{i}>0$,

$$
\left(\nabla K_{2}-\nabla K_{1}\right) \cdot N_{0} \leq 0 \text { on } \Gamma_{T},
$$

and if $u_{i}$ is in $C^{1} \overline{\left(G_{T}^{i}\right)}$, then

$$
u_{i}<\kappa \text { on } \Gamma_{T}
$$

Proof. It suffices to prove that

$$
U_{i}<0 \text { in } \overline{G_{T}^{i}} .
$$

If the assertion is not true then, since $U_{i}<0$ in $\overline{G_{t_{*}}^{i}}$ if $t_{*}$ is small, there exists a $t_{0}>0$ such that $U_{i}<0$ in $\overline{G_{t}^{i}}$ for all $t<t_{0}$ but $U_{i}\left(x_{0}, t_{0}\right)=0$ for some $\left(x_{0}, t_{0}\right) \in \overline{G_{t_{0}}^{i}}$ where $i=1$ or $i=2$. By the maximum principle we deduce that $\left(x_{0}, t_{0}\right)$ must belong to $\Gamma_{T}$, and

$$
\begin{aligned}
U_{1}\left(x_{0}, t_{0}\right) & =U_{2}\left(x_{0}, t_{0}\right)=0, \\
\frac{d}{d t} U_{1} \geq 0, \quad \frac{d}{d s_{j}} U_{1} & =0, \quad\left(\nabla U_{1}-\nabla U_{2}\right) \cdot N_{0}>0
\end{aligned}
$$


at $\left(x_{0}, t_{0}\right)$. But then the last equation in (9.2) implies that $\left(\nabla K_{1}-\nabla \dot{K}_{2}\right) \cdot N_{0}>0$ at $\left(x_{0}, t_{0}\right)$, a contradiction to (9.3).

Consider the radial case where

$$
G=\{\alpha<|x|<\beta\}, \quad \tilde{\theta}=\tilde{\theta}(r, t), \quad r=|x|,
$$

with the free boundary condition

$$
\theta=\frac{\varepsilon}{s(t)}, \quad-\theta_{1, r}+\theta_{2, r}=\frac{d s}{d t} .
$$

If $n \geq 3$ then taking $K_{1}=K_{2}=1 / r$ we have

$$
\partial_{t} K_{i}-\Delta K_{i}=\frac{n-3}{r^{3}} \geq 0 \text {. }
$$

Since also $u_{i}$ is in $C^{1}\left(\overline{G_{T}^{i}}\right)$ is in this case (the proof is similar to that of Theorem 6.1), we conclude

Theorem 9.2. In the radial case, if $n \geq 3$, then (9.4) holds, i.e., small surface tension decreases the water region for all $0 \leq t \leq T$.

Notice that (still in the radial case), if $K_{i}^{0}$ is the solution of (9.1) satisfying

$$
\partial_{t} K_{i}^{0}-\Delta K_{i}^{0}=0 \quad \text { on } G_{T}^{i}
$$

and

$$
0<l_{i} \equiv l_{i}^{0}<\frac{1}{r}-\delta \quad \text { on } \partial D_{T}^{i} \cup\left(G_{i} \times\{0\}\right)
$$

where $0<\delta$ is sufficiently small, then, by comparison with $K_{i}=\frac{1}{r}$, there exists $\varepsilon=\varepsilon(\delta, T)>0$ such that

$$
\left(\nabla K_{1}^{0}-\nabla K_{2}^{0}\right) \cdot N_{0}>\varepsilon \quad \text { on } \Gamma_{T} .
$$

It then follows that for the nonradial case, if $n \geq 3$ and the data are "close" to radial, we can establish (9.4) for a smooth approximation of $u_{i}$ (as constructed in Remark 8.1). Hence $u_{i} \leq \kappa$ on $\Gamma_{T}$.

Consider finally the radial case with $n=2$; we further specialize to a free boundary $|x|=t^{1 / 2} \quad(t \geq 1)$, which corresponds to

$$
\theta_{0, i}=f_{i}\left(\frac{|x|}{t^{1 / 2}}\right)=\alpha_{i} \int_{|x| / t^{1 / 2}}^{\infty} \zeta^{-1} e^{-\zeta^{2} / 4} d \zeta+\beta_{i}
$$

with appropriate $\alpha_{i}, \beta_{i}$.

Introducing

$$
W_{i}(y, s)=y e^{s / 2} K_{i}\left(y e^{s / 2}, e^{s}\right)
$$

we find that the system of equations for the $K_{i}$ becomes

$$
\mathscr{L} W_{i} \equiv \frac{\partial W_{i}}{\partial s}-\frac{\partial^{2} W_{i}}{\partial y^{2}}-\left(\frac{y}{2}-\frac{1}{y}\right) \frac{\partial W_{i}}{\partial y}-\frac{W_{i}}{y^{2}} \geq 0 \quad \text { in } \Omega_{T}^{i}
$$


with initial and boundary conditions

$$
\begin{array}{ll}
W_{i}=1 & \text { for } y=1, \\
W_{i}>0 & \text { on the remaining part of } \partial \Omega_{T}^{i}
\end{array}
$$

where

$$
\begin{aligned}
& \Omega_{T}^{1}=\left\{(y, s) ; \alpha e^{-s / 2}<y<1,0<s<T\right\}, \\
& \Omega_{T}^{2}=\left\{(y, s) ; 1<y<\beta e^{-s / 2}, 0<s<T\right\} \quad(0<\alpha<\beta<\infty) .
\end{aligned}
$$

Let $\widehat{W}_{i}$ be the solution of $\mathscr{L} \widehat{W}_{i}=0$ in $\widehat{\Omega}_{T}^{i}$ where

$$
\begin{aligned}
& \widehat{\Omega}_{T}^{1}=\left\{(y, s) ; \alpha e^{-T / 2}<y<1,0<s<T\right\} \supset \Omega_{T}^{1}, \\
& \widehat{\Omega}_{T}^{2}=\{(y, s) ; 1<y<\beta, 0<s<T\} \supset \Omega_{T}^{2},
\end{aligned}
$$

satisfying the initial and boundary conditions:

$$
\begin{array}{ll}
\widehat{W}_{i}=1 & \text { for } y=1, \\
\widehat{W}_{1}=0 & \text { if } y=\alpha e^{-T / 2}, \\
\widehat{W}_{2}=0 & \text { if } y=\beta, \\
\widehat{W}_{i}=0 & \text { if } s=0 .
\end{array}
$$

Then, by the maximum principle, $W_{i}>\widehat{W}_{i}$ in $\Omega_{T}^{i}$ (if we choose $W_{i}=\widehat{W}_{i}$ on the parabolic part of $\Omega_{T}^{i}$ ) and

$$
\frac{\partial \widehat{W}_{2}}{\partial y}-\frac{\partial \widehat{W}_{1}}{\partial y}>\frac{\partial W_{2}}{\partial y}-\frac{\partial W_{1}}{\partial y} \text { for } y=1 \text {. }
$$

Applying the maximum principle to $\partial \widehat{W}_{i} / \partial s$ we deduce that

$$
\frac{\partial \widehat{W}_{i}}{\partial s} \geq 0 \text {. }
$$

We now integrate by parts in

$$
\int_{\alpha \varepsilon^{-T / 2}}^{1} \mathscr{L} \widehat{W}_{1}(y, s) d y=0, \quad \int_{1}^{\beta} \mathscr{L} \widehat{W}_{2}(y, s) d y=0
$$

and add the results; we obtain

$$
\begin{aligned}
\widehat{W}_{1, y}(1, s)-\widehat{W}_{2, y}(1, s)= & \int_{\alpha e^{-T / 2}}^{1}\left(\partial_{s} \widehat{W}_{1}+\frac{1}{2} \widehat{W}_{1}\right)+\int_{1}^{\beta}\left(\partial_{s} \widehat{W}_{2}+\frac{1}{2} \widehat{W}_{2}\right) \\
& +\left[\widehat{W}_{1, y}\left(\alpha e^{-T / 2}, s\right)-\widehat{W}_{2, y}(\beta, s)\right] .
\end{aligned}
$$

Recalling (9.8) and noting that the last expression in brackets is positive (by the maximum principle), it follows that

$$
\widehat{W}_{1, y}(1, s)-\widehat{W}_{2, y}(1, s)>0,
$$

and from (9.8), (9.5) we then deduce that the condition (9.3) is satisfied. Therefore, by Lemma 9.1: 
Theorem 9.3. If $n=2$ and the free boundary is given by $|x|=t^{1 / 2}$ then (9.4) holds for all $T>0$, i.e., small surface tension decreases the water region for all $t \geq 0$.

Remark 9.1. One can extend Theorem 9.3 also to other radial free boundaries. For instance, if the free boundary has the form

$$
|x|=s(t) \quad \text { where } \dot{s}(t) \leq 0 \text { for } 0<t<T
$$

then, by choosing

$$
K_{1}(x, t)=\frac{1}{s(t)} \quad \text { and } \quad K_{2}(x, t)=\frac{1}{s(t)},
$$

the conditions of Lemma 9.1 are satisfied; consequently (9.4) holds for all $0<$ $t \leq T$.

\section{REFERENCES}

1. G. Caginalp, An analysis of a phase field model of a free boundary, Arch. Rational Mech. Anal. 92 (1986), 205-245.

2. B. Chalmers, Principles of solidification, Krieger, Huntington, N.Y., 1977.

3. J. Duchon and R. Robert, Évolution d'une interface par capillarité et diffusion de volume I. Existence locale en temps, Ann. Inst. Henri Poincaré 1 (1984), 361-378.

4. A. Friedman, Remarks on the maximum principle for parabolic equations and its applications, Pacific J. Math. 8 (1958), 201-211.

5. __, Partial differential equations of parabolic type, Prentice-Hall, Englewood Cliffs, N.J., 1964.

6. __ Variational principles and free-boundary problem, Wiley-Interscience, New York, 1982.

7. A. Friedman and D. Kinderlehrer, A one-phase Stefan problems, Indiana Univ. Math. J. 24 (1975), 1005-1035.

8. J. W. Gibbs, Collected Works, Yale Univ. Press, New Haven, Conn., 1948.

9. M. E. Gurtin, On the two phase Stefan problem with interfacial energy and entropy, Arch. Rational Mech. Anal. 96 (1986), 199-241.

10. __ Multiphase thermomechanics with interfacial structure 1. Heat conduction and the capillary balance law, Arch. Rational Mech. Anal. 104 (1988), 195-221.

11. E. Hanzawa, Classical solution of the Stefan problem, Tôhoku Math. J. 33 (1981), 297-335.

12. P. Hartman, Crystal growth: An introduction, North-Holland, Amsterdam, 1973.

13. O. A. Ladyzenskaja, V. A. Solonnikov and N. N. Ural' ceva, Linear and quasilinear equations of parabolic type, Amer. Math. Soc. Transl., Amer. Math. Soc., Providence, R.I., 1968.

14. J. S. Langer, Instabilities and pattern formation in crystal growth, Rev. Mod. Phys. 52 (1980), $1-28$.

15. H. Matano, Asymptotic behavior of the free boundaries arising in one phase Stefan problems in multi-dimensional spaces, Lecture Notes in Numer. Appl. Anal., vol. 5, Kinokuniya, Tokyo, 1982, pp. 133-151.

16. A. M. Meirmanov, On a classical solution of the multidimensional Stefan problem for quasilinear parabolic equations, Math. Sb. 112 (1980), 170-192.

17. W. W. Mullins, Thermodynamic equilibrium of a crystal sphere in a fluid, J. Chem. Phys. 81 (1984), 1436-1442.

18. W. W. Mullins and R. F. Sekerka, Stability of a planar interface during solidification of a dilute binary alloy, J. Appl. Phys. 35 (1964), 444-451. 
19. A. Visintin, Models for supercooling and superheating effects, Research Notes in Math., no. 120, Pitman, 1985, pp. 200-207.

20. S. Luckhaus, Solutions for the two-phase Stefan problem with the Gibbs-Thomsom law for melting temperature, Europ. J. Appl. Math. 1 (1990), 101-111.

Institute for Mathematics and its Applications, University of Minnesota, MinneapoLis, MinNeSOTA 55455

School of Mathematics, University of Minnesota, Minneapolis, Minnesota 55455 